\title{
Monodromy of a Class of Logarithmic Connections on an Elliptic Curve
}

\author{
Francois-Xavier MACHU
}

Mathématiques - bât. M2, Université Lille 1, F-59655 Villeneuve d'Ascq Cedex, France

E-mail: xavier.machu@math.univ-lille1.fr

Received March 22, 2007, in final form August 06, 2007; Published online August 16, 2007

Original article is available at http://www.emis.de/journals/SIGMA/2007/082/

\begin{abstract}
The logarithmic connections studied in the paper are direct images of regular connections on line bundles over genus-2 double covers of the elliptic curve. We give an explicit parametrization of all such connections, determine their monodromy, differential Galois group and the underlying rank-2 vector bundle. The latter is described in terms of elementary transforms. The question of its (semi)-stability is addressed.
\end{abstract}

Key words: elliptic curve; ramified covering; logarithmic connection; bielliptic curve; genus-2 curve; monodromy; Riemann-Hilbert problem; differential Galois group; elementary transformation; stable bundle; vector bundle

2000 Mathematics Subject Classification: 14D21; 14H52; 14H60; 32S40

\section{Introduction}

The Riemann-Hilbert correspondence relates the integrable logarithmic (or Fuchsian) connections over an algebraic variety $X$ to the representations of the fundamental group $\pi_{1}(X \backslash\{D\})$, where $D$ denotes the divisor of poles of a connection. Deligne [3] proved its bijectivity, on condition that $D$ is a fixed normal crossing divisor and the data on both sides are taken modulo appropriate equivalence relations. Nevertheless, Deligne's solution is not effective in the sense that it does not imply any formulas to compute the Riemann-Hilbert correspondence. Therefore, it is important to have on hand a stock of examples that can be solved explicitly.

The authors of $[13,5]$ constructed logarithmic connections of rank $n$ over $\mathbb{P}^{1}$ with quasipermutation monodromy in terms of theta functions on a ramified cover of $\mathbb{P}^{1}$ of degree $n$. Korotkin in [12] considers a class of generalized connections, called connections with constant twists, and constructs such twisted connections of rank 2 with logarithmic singularities on an elliptic curve $E$ via theta functions on a double cover $C$ of $E$.

In the present paper, we obtain genuine (non-twisted) rank-2 connections on $E$ from its double cover $C$ by a different method, similar to the method applied in [15] to the double covers of $\mathbb{P}^{1}$. We consider a genus-2 cover $f: C \rightarrow E$ of degree 2 with two branch points $p_{+}, p_{-}$and a regular connection $\nabla_{\mathcal{L}}$ on a line bundle $\mathcal{L}$ over $C$. Then the sheaf-theoretic direct image $\mathcal{E}=f_{*}(\mathcal{L})$ is a rank- 2 vector bundle carrying the connection $\nabla_{\mathcal{E}}:=f_{*}\left(\nabla_{\mathcal{L}}\right)$ with logarithmic poles at $p_{+}$ and $p_{-}$. We explicitly parameterize all such connections and their monodromy representations $\rho: \pi_{1}\left(E \backslash\left\{p_{-}, p_{+}\right\}\right) \rightarrow G L(2, \mathbb{C})$. We also investigate the abstract group-theoretic structure of the obtained monodromy groups as well as their Zariski closures in $G L(2, \mathbb{C})$, which are the differential Galois groups of the connections $\nabla_{\mathcal{E}}$.

Establishing a bridge between the analytic and algebro-geometric counterparts of the problem is one of the main objectives of the paper. We show that the underlying vector bundle $\mathcal{E}$ of $\nabla_{\mathcal{E}}$ is stable of degree -1 for generic values of parameters and identify the special cases where it is unstable and is the direct sum of two line bundles. 
We also illustrate the following Bolibruch-Esnault-Viehweg Theorem [7]: any irreducible logarithmic connection over a curve can be converted by a sequence of Gabber's transforms into a logarithmic connection with same singularities and same monodromy on a semistable vector bundle of degree 0 . Bolibruch has established this result in the genus-0 case, in which "semistable of degree 0" means just "trivial" [1].

We explicitly indicate a Gabber's transform of the above direct image connection $\left(\mathcal{E}, \nabla_{\mathcal{E}}\right)$ which satisfies the conclusion of the Bolibruch-Esnault-Viehweg Theorem. The importance of results of this type is that they allow us to consider maps from the moduli space of connections to the moduli spaces of vector bundles, for only semistable bundles have a consistent moduli theory. Another useful feature of the elementary transforms is that they permit to change arbitrarily the degree, and this enriches our knowledge of the moduli space of connections providing maps to moduli spaces of vector bundles of different degrees, which may be quite different and even have different dimensions (see Remark 2).

All the relevant algebro-geometric tools are introduced in a way accessible to a non-specialist. One of them is the usage of ruled surfaces in finding line subbundles of rank-2 vector bundles. This is classical, see [14] and references therein. Another one is the reconstruction of a vector bundle from the singularities of a given connection on it. Though it is known as a theoretical method [6, 7], it has not been used for a practical calculation of vector bundles underlying a given meromorphic connection over a Riemann surface different from the sphere. For the Riemann sphere, any vector bundle is the direct sum of the line bundles $\mathcal{O}\left(k_{i}\right)$, and Bolibruch developed the method of valuations (see [1]) serving to calculate the integers $k_{i}$ for the underlying vector bundles of connections. He exploited extensively this method, in particular in his construction of counter-examples to the Riemann-Hilbert problem for reducible representations.

Genus-2 double covers of elliptic curves is a classical subject, originating in the work of Legendre and Jacobi [9]. We provide several descriptions of them, based on a more recent work [4]. We determine the locus of their periods (Corollary 1), a result which we could not find elsewhere in the literature and which we need for finding the image of the Riemann-Hilbert correspondence in Proposition 5.

Now we will briefly survey the contents of the paper by sections. In Section 2, we describe the genus- 2 covers of elliptic curves of degree 2 and determine their periods. In Section 3 , we investigate rank-1 connections on $C$ and discuss the dependence of the Riemann-Hilbert correspondence for these connections on the parameters of the problem: the period of $C$ and the underlying line bundle $\mathcal{L}$. In Section 4 , we compute, separately for the cases $\mathcal{L}=\mathcal{O}_{C}$ and $\mathcal{L} \neq \mathcal{O}_{C}$, the matrix of the direct image connection $\nabla_{\mathcal{E}}$ on $\mathcal{E}=f_{*} \mathcal{L}$. For $\mathcal{L}=\mathcal{O}_{C}$, we also provide two different forms for a scalar ODE of order 2 equivalent to the $2 \times 2$ matrix equation $\nabla_{\mathcal{E}} \varphi=0$. In Section 5, we determine the fundamental matrices and the monodromy of connections $\nabla_{\mathcal{E}}$ and discuss their isomonodromy deformations. Section 6 introduces the elementary transforms of rank-2 vector bundles, relates them to birational maps between ruled surfaces and states a criterion for (semi)-stability of a rank-2 vector bundle. In Section 7, we apply the material of Section 6 to describe $\mathcal{E}$ as a result of a series of elementary transforms starting from $\mathcal{E}_{0}=f_{*} \mathcal{O}_{C}$ and prove its stability or unstability depending on the value of parameters. We also describe Gabber's elementary transform which illustrates the BolibruchEsnault-Viehweg Theorem and comment briefly on the twisted connections of [12]. In Section 8 , we give a description of the structure of the monodromy and differential Galois groups for $\nabla_{\mathcal{E}}$.

Terminology. If not specified otherwise, a curve will mean a nonsingular complex projective algebraic curve, which we will not distinguish from the associated analytic object, a compact Riemann surface. 


\section{Genus-2 covers of an elliptic curve}

In this section, we will describe the degree-2 covers of elliptic curves which are curves of genus 2 .

Definition 1. Let $\pi: C \rightarrow E$ be a degree-2 map of curves. If $E$ is elliptic, then we say that $C$ is bielliptic and that $E$ is a degree-2 elliptic subcover of $C$. form

Legendre and Jacobi [9] observed that any genus-2 bielliptic curve has an equation of the

$$
y^{2}=c_{0} x^{6}+c_{1} x^{4}+c_{2} x^{2}+c_{3} \quad\left(c_{i} \in \mathbb{C}\right)
$$

in appropriate affine coordinates $(x, y)$. It immediately follows that any bielliptic curve $C$ has two elliptic subcovers $\pi_{i}: C \rightarrow E_{i}$,

$$
\begin{aligned}
& E_{1}: y^{2}=c_{0} x_{1}^{3}+c_{1} x_{1}^{2}+c_{2} x_{1}+c_{3}, \quad \pi_{1}:(x, y) \mapsto\left(x_{1}=x^{2}, y\right), \quad \text { and } \\
& E_{2}: \quad y_{2}^{2}=c_{3} x_{2}^{3}+c_{2} x_{2}^{2}+c_{1} x_{2}+c_{0}, \quad \pi_{2}:(x, y) \mapsto\left(x_{2}=1 / x^{2}, y_{2}=y / x^{3}\right) .
\end{aligned}
$$

This description of bielliptic curves, though very simple, depends on an excessive number of parameters. To eliminate unnecessary parameters, we will represent $E_{i}$ in the form

$$
E_{i}: y_{i}^{2}=x_{i}\left(x_{i}-1\right)\left(x_{i}-t_{i}\right) \quad\left(t_{i} \in \mathbb{C} \backslash\{0,1\}, t_{1} \neq t_{2}\right) .
$$

Remark that any pair of elliptic curves $\left(E_{1}, E_{2}\right)$ admits such a representation even if $E_{1} \simeq E_{2}$.

We will describe the reconstruction of $C$ starting from $\left(E_{1}, E_{2}\right)$ following [4]. This procedure will allow us to determine the periods of bielliptic curves $C$ in terms of the periods of their elliptic subcovers $E_{1}, E_{2}$.

Let $\varphi_{i}: E_{i} \rightarrow \mathbb{P}^{1}$ be the double cover map $\left(x_{i}, y_{i}\right) \mapsto x_{i}(i=1,2)$. Recall that the fibered product $E_{1} \times_{\mathbb{P}^{1}} E_{2}$ is the set of pairs $\left(P_{1}, P_{2}\right) \in E_{1} \times E_{2}$ such that $\varphi_{1}\left(P_{1}\right)=\varphi_{2}\left(P_{2}\right)$. It can be given by two equations with respect to three affine coordinates $\left(x, y_{1}, y_{2}\right)$ :

$$
\bar{C}:=E_{1} \times_{\mathbb{P}^{1}} E_{2}:\left\{\begin{array}{l}
y_{1}^{2}=x(x-1)\left(x-t_{1}\right), \\
y_{2}^{2}=x(x-1)\left(x-t_{2}\right) .
\end{array}\right.
$$

It is easily verified that $\bar{C}$ has nodes over the common branch points $0,1, \infty$ of $\varphi_{i}$ and is nonsingular elsewhere. For example, locally at $x=0$, we can choose $y_{i}$ as a local parameter on $E_{i}$, so that $x$ has a zero of order two on $E_{i}$; equivalently, we can write $x=f_{i}\left(y_{i}\right) y_{i}^{2}$ where $f_{i}$ is holomorphic and $f_{i}(0) \neq 0$. Then eliminating $x$, we obtain that $\bar{C}$ is given locally by a single equation $f_{1}\left(y_{1}\right) y_{1}^{2}=f_{2}\left(y_{2}\right) y_{2}^{2}$. This is the union of two smooth transversal branches $\sqrt{f_{1}\left(y_{1}\right)} y_{1}= \pm \sqrt{f_{2}\left(y_{2}\right)} y_{2}$.

Associated to $\bar{C}$ is its normalization (or desingularization) $C$ obtained by separating the two branches at each singular point. Thus $C$ has two points over $x=0$, whilst the only point of $\bar{C}$ over $x=0$ is the node, which we will denote by the same symbol 0 . We will also denote by $0_{+}, 0_{-}$ the two points of $C$ over 0 . Any of the functions $y_{1}, y_{2}$ is a local parameter at $0_{ \pm}$. In a similar way, we introduce the points $1, \infty \in \bar{C}$ and $1_{ \pm}, \infty_{ \pm} \in C$.

Proposition 1. Given a genus-2 bielliptic curve $C$ with its two elliptic subcovers $\pi_{i}: C \rightarrow E_{i}$, one can choose affine coordinates for $E_{i}$ in such a way that $E_{i}$ are given by the equations (3), $C$ is the normalization of the nodal curve $\bar{C}:=E_{1} \times_{\mathbb{P}^{1}} E_{2}$, and $\pi_{i}=\operatorname{pr}_{i} \circ \nu$, where $\nu: C \rightarrow \bar{C}$ denotes the normalization map and $\mathrm{pr}_{i}$ the projection onto the $i$-th factor.

Proof. See [4]. 
It is interesting to know, how the descriptions given by (1) and Proposition 1 are related to each other. The answer is given by the following proposition.

Proposition 2. Under the assumptions and in the notation of Proposition 1, apply the following changes of coordinates in the equations of the curves $E_{i}$ :

$$
\left(x_{i}, y_{i}\right) \rightarrow\left(\tilde{x}_{i}, \tilde{y}_{i}\right), \quad \tilde{x}_{i}=\frac{x_{i}-t_{j}}{x_{i}-t_{i}}, \quad \tilde{y}_{i}=\frac{y_{i}}{\left(x_{i}-t_{i}\right)^{2}} \sqrt{\frac{\left(t_{j}-t_{i}\right)^{3}}{t_{i}\left(1-t_{i}\right)}},
$$

where $j=3-i, i=1,2$, so that $\{i, j\}=\{1,2\}$. Then the equations of $E_{i}$ acquire the form

$$
\begin{aligned}
& E_{1}: \quad \tilde{y}_{1}^{2}=\left(\tilde{x_{1}}-\frac{t_{2}}{t_{1}}\right)\left(\tilde{x_{1}}-\frac{1-t_{2}}{1-t_{1}}\right)\left(\tilde{x_{1}}-1\right), \\
& E_{2}: \quad \tilde{y}_{2}^{2}=\left(1-\frac{t_{2}}{t_{1}} \tilde{x_{2}}\right)\left(1-\frac{1-t_{2}}{1-t_{1}} \tilde{x_{2}}\right)\left(1-\tilde{x_{2}}\right) .
\end{aligned}
$$

Further, $C$ can be given by the equation

$$
\eta^{2}=\left(\xi^{2}-\frac{t_{2}}{t_{1}}\right)\left(\xi^{2}-\frac{1-t_{2}}{1-t_{1}}\right)\left(\xi^{2}-1\right)
$$

and the maps $\pi_{i}: C \rightarrow E_{i}$ by $(\xi, \eta) \mapsto\left(\tilde{x}_{i}, \tilde{y}_{i}\right)$, where

$$
\left(\tilde{x}_{1}, \tilde{y}_{1}\right)=\left(\xi^{2}, \eta\right), \quad\left(\tilde{x}_{2}, \tilde{y}_{2}\right)=\left(1 / \xi^{2}, \eta / \xi^{3}\right) .
$$

Proof. We have the following commutative diagram of double cover maps

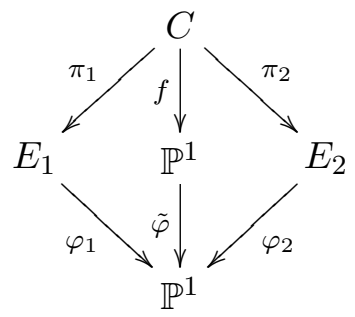

in which the branch loci of $\tilde{\varphi}, \varphi_{i}, f, \pi_{i}$ are respectively $\left\{t_{1}, t_{2}\right\},\left\{0,1, t_{i}, \infty\right\}, \tilde{\varphi}^{-1}(\{0,1, \infty\})$, $\varphi_{i}^{-1}\left(t_{j}\right)(j=3-i)$. Thus the $\mathbb{P}^{1}$ in the middle of the diagram can be viewed as the Riemann surface of the function $\sqrt{\frac{x-t_{2}}{x-t_{1}}}$, where $x$ is the coordinate on the bottom $\mathbb{P}^{1}$. We introduce a coordinate $\xi$ on the middle $\mathbb{P}^{1}$ in such a way that $\tilde{\varphi}$ is given by $\xi \mapsto x, \xi^{2}=\frac{x-t_{2}}{x-t_{1}}$. Then $C$ is the double cover of $\mathbb{P}^{1}$ branched in the 6 points $\tilde{\varphi}^{-1}(\{0,1, \infty\})=\left\{ \pm 1, \pm \sqrt{\frac{1-t_{2}}{1-t_{1}}}, \pm \sqrt{\frac{t_{2}}{t_{1}}}\right\}$, which implies the equation (5) for $C$. Then we deduce the equations of $E_{i}$ in the form (4) following the recipe of (2), and it is an easy exercise to transform them into (3).

The locus of bielliptic curves in the moduli space of all the genus-2 curves is 2-dimensional, hence is a hypersurface. In [16], an explicit equation of this hypersurface is given in terms of the Igusa invariants of the genus- 2 curves. We will give a description of the same locus in terms of periods. We start by recalling necessary definitions.

Let $a_{1}, a_{2}, b_{1}, b_{2}$ be a symplectic basis of $H_{1}(C, \mathbb{Z})$ for a genus- 2 curve $C$, and $\omega_{1}, \omega_{2}$ a basis of the space $\Gamma\left(C, \Omega_{C}^{1}\right)$ of holomorphic 1-forms on $C$.

Definition 2. Let us introduce the $2 \times 2$-matrices $A=\left(\int_{a_{i}} \omega_{j}\right)$ and $B=\left(\int_{b_{i}} \omega_{j}\right)$. Their concatenation $\Pi=(A \mid B)$ is a $2 \times 4$ matrix, called the period matrix of the 1 -forms $\omega_{1}$, $\omega_{2}$ with respect to the basis $a_{1}, a_{2}, b_{1}, b_{2}$ of $H_{1}(C, \mathbb{Z})$. The period of $C$ is the $2 \times 2$-matrix $Z=A^{-1} B$. If 


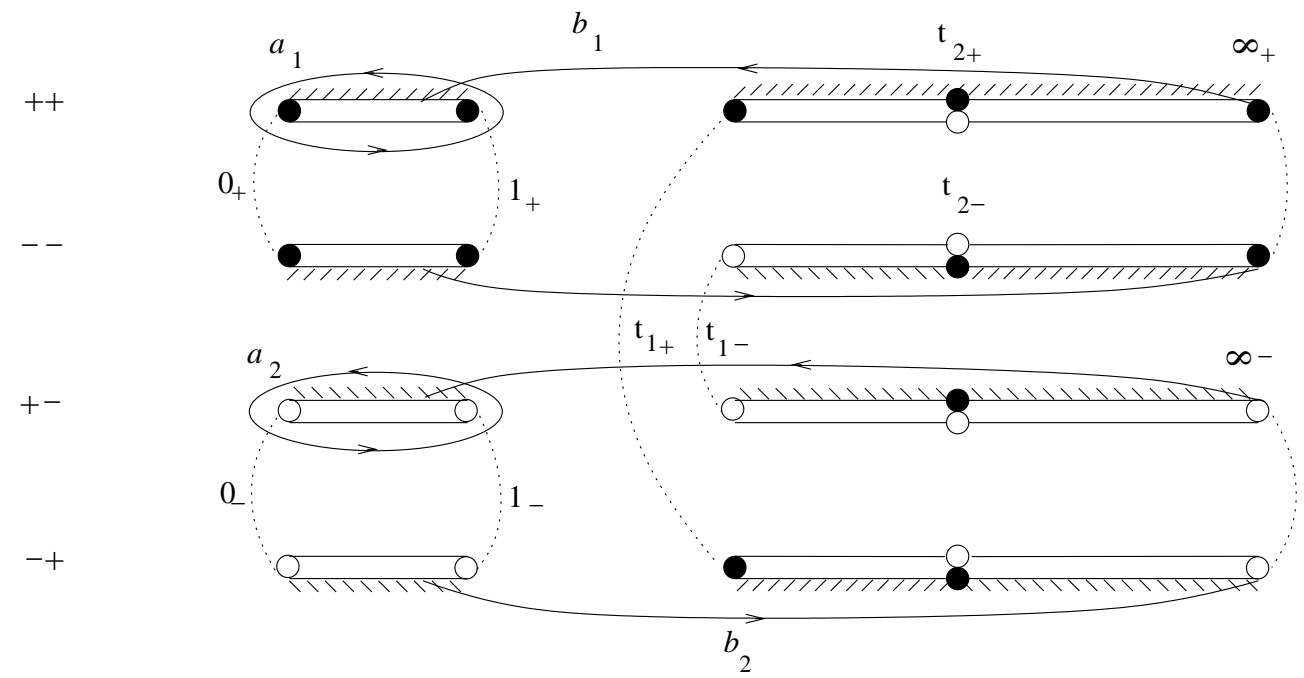

Figure 1. The 4 sheets of $C$. The segments of two edges of the cuts are glued together if they are: (1) situated one under the other, and (2) hatched by dashes of the same orientation. Thus, the upper edge of the cut on $\Sigma_{++}$between $t_{1}, t_{2}$ is glued to the lower edge of the cut on $\Sigma_{-+}$between $t_{1}, t_{2}$. Four black points over $t_{2}$ glue together to give one point $t_{2+} \in C$, and similarly four white ones give $t_{2-} \in C$. The 4 preimages of each one of the points $0,1, t_{1}, \infty$ are glued in pairs, as shown by the colors black/white and by dotted lines, and give 8 points of $C$ denoted by $0_{ \pm}, 1_{ \pm}, t_{1 \pm}, \infty_{ \pm}$.

$A=I$ is the identity matrix, the basis $\omega_{1}, \omega_{2}$ of $\Gamma\left(C, \Omega_{C}^{1}\right)$ and the corresponding period matrix $\Pi_{0}=(I \mid Z)$ are called normalized.

The period lattice $\Lambda=\Lambda(C)$ is the $\mathbb{Z}$-submodule of rank 4 in $\Gamma\left(C, \Omega_{C}^{1}\right)^{*}$ generated by the 4 linear forms $\omega \mapsto \int_{a_{i}} \omega, \omega \mapsto \int_{b_{i}} \omega$. A choice of the basis $\omega_{i}$ identifies $\Gamma\left(C, \Omega_{C}^{1}\right)^{*}$ with $\mathbb{C}^{2}$, and $\Lambda$ is then generated by the 4 columns of $\Pi$.

The period $Z_{C}$ of $C$ is determined modulo the discrete group $\operatorname{Sp}(4, \mathbb{Z})$ acting by symplectic base changes in $H_{1}(C, \mathbb{Z})$.

Riemann's bilinear relations. The period matrix of any genus- 2 curve $C$ satisfies the conditions

$$
Z^{t}=Z \quad \text { and } \quad \Im Z>0 .
$$

To determine the periods of bielliptic curves $C$, it is easier to use the representation from Proposition 1 rather than the standard equation of a genus- 2 curve (5). This is due to the fact that we can choose $\omega_{1}=d x / y_{1}, \omega_{2}=d x / y_{2}$ as a basis of the space $\Gamma\left(C, \Omega_{C}^{1}\right)$ of holomorphic 1-forms on $C$, and the periods of these 1-forms are easily related to the periods on $E_{i}$. (Basically, $\left(\omega_{1}, \omega_{2}\right)$ can be seen as a basis of eigenvectors of the action of $(\mathbb{Z} / 2 \mathbb{Z})^{2}$ on $C$.)

To fix the ideas, we assume for a while that $t_{1}, t_{2}$ are real and $1<t_{1}<t_{2}$ (the general case is obtained by a deformation moving the points $t_{i}$ ). $E_{i}$ can be represented as the result of gluing two sheets $\Sigma_{i+}, \Sigma_{i-}$, which are Riemann spheres with cuts along the segments $[0,1]$ and $\left[t_{i}, \infty\right]$. Then $C$, parameterizing the pairs of points $\left(P_{1}, P_{2}\right)$ with $P_{i} \in E_{i}$ and with the same $x$-coordinate, is the result of gluing 4 sheets, which are copies of the Riemann sphere with cuts along the segments $[0,1]$ and $\left[t_{1}, \infty\right]$ labelled by,,,++--+--+ . For example, the sheet $\Sigma_{+-}$is formed by the pairs $\left(P_{1}, P_{2}\right)$ where $P_{1}$ lies on $\Sigma_{1+}$ and $P_{2}$ on $\Sigma_{2-}$. Fig. 1 shows the gluings of the edges of the cuts with the help of hatching and fixes the choice of the cycles $a_{i}, b_{i}$. Black points on one vertical are identified, the same for the white ones. 
Proposition 3. Let $C, E_{1}, E_{2}$ be as in Proposition 1, and $a_{i}, b_{i}$ as on Fig. 1. Then the period matrix of $C$ is

$$
Z_{C}=\left(\begin{array}{cc}
\frac{1}{2}\left(\tau_{1}+\tau_{2}\right) & \frac{1}{2}\left(\tau_{1}-\tau_{2}\right) \\
\frac{1}{2}\left(\tau_{1}-\tau_{2}\right) & \frac{1}{2}\left(\tau_{1}+\tau_{2}\right)
\end{array}\right)
$$

where $\tau_{i}$ is the period of $E_{i}$ with respect to the basis $\gamma_{i}=\pi_{i *}\left(a_{1}\right), \delta_{i}=\pi_{i *}\left(b_{1}\right)$ of $H_{1}\left(E_{i}, \mathbb{Z}\right)$.

Proof. Let $k_{i}, l_{i}$ be the periods of the differential $d x / y_{i}$ on $E_{i}$ along the cycles $\gamma_{i}, \delta_{i}$ respectively. Take $\omega_{i}=\pi_{i}^{*}\left(d x / y_{i}\right)$ as a basis of $\Gamma\left(C, \Omega_{C}\right)$. We have

$$
\int_{a_{1}} \pi_{j}^{*}\left(d x / y_{j}\right)=\int_{\pi_{j *}\left(a_{1}\right)} d x / y_{j}=k_{j} .
$$

But when calculating the integral over $a_{2}$, we have to take into account the fact that a positively oriented loop around a cut on $\Sigma_{+-}$projects to a positively oriented loop on $\Sigma_{2-}$, and the latter defines the cycle $-\gamma_{2}$ on $E_{2}$. Thus $\pi_{2 *}\left(a_{2}\right)=-\gamma_{2}$, and the corresponding period acquires an extra sign:

$$
\int_{a_{2}} \pi_{j}^{*}\left(d x / y_{j}\right)=\int_{\pi_{j *}\left(a_{2}\right)} d x / y_{j}=(-1)^{j+1} k_{j}
$$

The integrals over $b_{j}$ are transformed in a similar way. We obtain the period matrix of $C$ in the form

$$
\Pi=\left(\begin{array}{cc|cc}
k_{1} & k_{1} & l_{1} & l_{1} \\
k_{2} & -k_{2} & l_{2} & -l_{2}
\end{array}\right) .
$$

Multiplying by the inverse of the left $2 \times 2$-block and using the relations $\tau_{i}=l_{i} / k_{i}$, we obtain the result.

Corollary 1. The locus $\mathcal{H}$ of periods of genus-2 curves $C$ with a degree-2 elliptic subcover is the set of matrices

$$
Z_{C}=\left(\begin{array}{cc}
\frac{1}{2}\left(\tau+\tau^{\prime}\right) & \frac{1}{2}\left(\tau-\tau^{\prime}\right) \\
\frac{1}{2}\left(\tau-\tau^{\prime}\right) & \frac{1}{2}\left(\tau+\tau^{\prime}\right)
\end{array}\right) \quad\left(\Im \tau>0, \Im \tau^{\prime}>0\right) .
$$

Equivalently, $\mathcal{H}$ is the set of all the matrices of the form $Z=\left(\begin{array}{ll}a & b \\ b & a\end{array}\right)(a, b \in \mathbb{C})$ such that $\Im Z>0$.

\section{Rank-1 connections on $C$ and their monodromy}

We start by recalling the definition of a connection. Let $V$ be a curve or a complement of a finite set in a curve $C$. Let $\mathcal{E}$ be a vector bundle of rank $r \geq 1$ on $V$. We denote by $\mathcal{O}_{V}, \Omega_{V}^{1}$ the sheaves of holomorphic functions and 1-forms on $V$ respectively. By abuse of notation, we will denote in the same way vector bundles and the sheaves of their sections. A connection on $\mathcal{E}$ is a $\mathbb{C}$-linear map of sheaves $\nabla: \mathcal{E} \rightarrow \mathcal{E} \otimes \Omega_{V}^{1}$ which satisfies the Leibnitz rule: for any open $U \subset V$, $f \in \Gamma(U, \mathcal{O})$ and $s \in \Gamma(U, \mathcal{E}), \nabla(f s)=f \nabla(s)+s d f$. If $\mathcal{E}$ is trivialized by a basis of sections $\boldsymbol{e}=\left(e_{1}, \ldots, e_{r}\right)$ over $U$, then we can write $\nabla\left(e_{j}\right)=\sum_{i} a_{i j} e_{i}$, and the matrix $A(\boldsymbol{e})=\left(a_{i j}\right)$ of holomorphic 1-forms is called the connection matrix of $\nabla$ with respect to the trivialization $e$. If there is no ambiguity with the choice of a trivialization, one can write, by abuse of notation, $\nabla=d+A$. 
Given $r$ meromorphic sections $s=\left(s_{1}, \ldots, s_{r}\right)$ which span $\mathcal{E}$ over an open subset, the matrix $A(s)$ defined as above is a matrix of meromorphic 1-forms on $V$. Its poles in $V$ are called apparent singularities of the connection with respect to the meromorphic trivialization $s$. The apparent singularities arise at the points $P \in V$ in which either some of the $s_{i}$ are non-regular, or all the $s_{i}$ are regular but $s_{i}(P)$ fail to be linearly independent. They are not singularities of the connection, but those of the chosen connection matrix.

In the case when the underlying vector bundle is defined not only over $V$, but over the whole compact Riemann surface $C$, we can speak about singularities at the points of $C \backslash V$ of the connection itself. To this end, choose local trivializations $\boldsymbol{e}_{P}$ of $\mathcal{E}$ at the points $P \in C \backslash V$, and define the local connection matrices $A\left(\boldsymbol{e}_{P}\right)$ as above, $\nabla\left(\boldsymbol{e}_{P}\right)=\boldsymbol{e}_{P} A\left(\boldsymbol{e}_{P}\right)$. The connection $\nabla$, regular on $V$, is said to be meromorphic on $C$ if $A\left(\boldsymbol{e}_{P}\right)$ has at worst a pole at $P$ for all $P \in C \backslash V$. If, moreover, $A\left(\boldsymbol{e}_{P}\right)$ can be represented in the form $A\left(\boldsymbol{e}_{P}\right)=B\left(\tau_{P}\right) \frac{d \tau_{P}}{\tau_{P}}$, where $\tau_{P}$ is a local parameter at $P$ and $B\left(\tau_{P}\right)$ is a matrix of holomorphic functions in $\tau_{P}$, then $P$ is said to be a logarithmic singularity of $\nabla$. A connection is called logarithmic, or Fuchsian, if it has only logarithmic singularities.

To define the monodromy of a connection $\nabla$, we have to fix a reference point $P_{0} \in V$ and a basis $s=\left(s_{1}, \ldots, s_{r}\right)$ of solutions of $\nabla s=0, s \in \Gamma(U, \mathcal{E})$ over a small disc $U$ centered at $P_{0}$. The analytic continuation of the $s_{i}$ along any loop $\gamma$ based at $P_{0}$ provides a new basis $\boldsymbol{s}^{\gamma}=\left(s_{1}^{\gamma}, \ldots, s_{r}^{\gamma}\right)$, and the monodromy matrix $M_{\gamma}$ is defined by $\boldsymbol{s}^{\gamma}=\boldsymbol{s} M_{\gamma}$. The monodromy matrix depends only on the homotopy class of a loop, and the monodromy $\rho_{\nabla}$ of $\nabla$ is the representation of the fundamental group of $V$ defined by

$$
\rho=\rho_{\nabla}: \pi_{1}\left(V, P_{0}\right) \longrightarrow G L_{r}(\mathbb{C}), \quad \gamma \mapsto M_{\gamma}
$$

Let now $C=V$ be a genus-2 bielliptic curve with an elliptic subcover $\varphi: C \rightarrow E$. Our objective is the study of rank-2 connections on $E$ which are direct images of rank-1 connections on $C$. We first study the rank- 1 connections on $C$ and their monodromy representations.

Let $\mathcal{L}$ be a line bundle on $C$ and $e$ a meromorphic section of $\mathcal{L}$ which is not identically zero. Then a connection $\nabla_{\mathcal{L}}$ on $\mathcal{L}$ can be written as $\mathrm{d}+\omega$, where $\omega$ is a meromorphic 1 -form on $C$ defined by $\nabla_{\mathcal{L}}(e)=\omega e$. The apparent singularities are simple poles with integer residues at the points where $e$ fails to be a basis of $\mathcal{L}$. We will start by considering the case when $\mathcal{L}$ is the trivial line bundle $\mathcal{O}=\mathcal{O}_{C}$. Then the natural trivialization of $\mathcal{L}$ is $e=1$, and $\omega$ is a regular 1-form. The vector space $\Gamma\left(C, \Omega_{C}^{1}\right)$ of regular 1 -forms on $C$ is 2-dimensional; let $\omega_{1}, \omega_{2}$ be its basis. We can write $\omega=\lambda_{1} \omega_{1}+\lambda_{2} \omega_{2}$ with $\lambda_{1}, \lambda_{2}$ in $\mathbb{C}$.

The horizontal sections of $\mathcal{O}$ are the solutions of the equation $\nabla_{\mathcal{O}} \varphi=0$. To write down these solutions, we can represent $C$ as in Proposition 1 and introduce the multi-valued functions $z_{1}=\int \omega_{1}$ and $z_{2}=\int \omega_{2}$, normalized by $z_{1}\left(\infty_{+}\right)=z_{2}\left(\infty_{+}\right)=0$. We denote by the same symbols $z_{1}, z_{2}$ the flat coordinates on the Jacobian $J C=\mathbb{C}^{2} / \Lambda$ associated to the basis $\left(\omega_{1}, \omega_{2}\right)$ of $\Gamma\left(C, \Omega_{C}^{1}\right)$, and $C$ can be considered as embedded in its Jacobian via the Abel-Jacobi map $A J: C \rightarrow J C, P \longmapsto\left(\left(z_{1}(P), z_{2}(P)\right)\right.$ modulo $\Lambda$.

To determine the monodromy, we will choose $P_{0}=\infty_{+}$and fix some generators $\alpha_{i}, \beta_{i}$ of $\pi_{1}\left(C, \infty_{+}\right)$in such a way that the natural epimorphism

$$
\pi_{1}\left(C, \infty_{+}\right) \longrightarrow H_{1}(C, \mathbb{Z})=\pi_{1}\left(C, \infty_{+}\right) /\left[\pi_{1}\left(C, \infty_{+}\right), \pi_{1}\left(C, \infty_{+}\right)\right]
$$

is given by $\alpha_{i} \mapsto a_{i}, \beta_{i} \mapsto b_{i}$.

The following lemma is obvious:

Lemma 1. The general solution of $\nabla_{\mathcal{O}} \varphi=0$ is given by $\varphi=c e^{-\lambda_{1} z_{1}-\lambda_{2} z_{2}}$, where $c$ is a complex constant. The monodromy matrices of $\nabla_{\mathcal{O}}$ are $M_{\alpha_{i}}=\exp \left(-\oint_{a_{i}} \omega\right), M_{\beta_{i}}=\exp \left(-\oint_{b_{i}} \omega\right)(i=$ $1,2)$. 
Now we turn to the problem of Riemann-Hilbert type: determine the locus of the representations of $G$ which are monodromies of connections $\nabla_{\mathcal{L}}$. Since any rank-1 representation $\rho$ of $G$ is determined by 4 complex numbers $\rho\left(\alpha_{i}\right), \rho\left(\beta_{i}\right)$, we can take $\left(\mathbb{C}^{*}\right)^{4}$ for the moduli space of representations of $G$ in which lives the image of the Riemann-Hilbert correspondence.

Before solving this problem on $C$, we will do a similar thing on an elliptic curve $E$. The answer will be used as an auxiliary result for the problem on $C$.

Any rank-1 representation $\rho: \pi_{1}(E) \rightarrow \mathbb{C}^{*}$ is determined by the images $\rho(a), \rho(b)$ of the generators $a, b$ of the fundamental group of $E$, so that the space of representations of $\pi_{1}(E)$ can be identified with $\mathbb{C}^{*} \times \mathbb{C}^{*}$. We will consider several spaces of rank-1 connections. Let $\mathcal{C}(E, \mathcal{L})$ be the space of all the connections $\nabla: \mathcal{L} \rightarrow \mathcal{L} \otimes \Omega_{E}^{1}$ on a line bundle $\mathcal{L}$ on $E$. It is non empty if only if $\operatorname{deg} \mathcal{L}=0$, and then $\mathcal{C}(E, \mathcal{L}) \simeq \Gamma\left(E, \Omega_{E}^{1}\right) \simeq \mathbb{C}$. Further, $\mathcal{C}(E)$ will denote the moduli space of pairs $(\mathcal{L}, \nabla)$, that is, $\mathcal{C}(E)=\cup_{[\mathcal{L}] \in J(E)} \mathcal{C}(E, \mathcal{L})$. We will also define the moduli space $\mathcal{C}$ of triples $\left(E_{\tau}, \mathcal{L}, \nabla\right), \mathcal{C}=\cup_{\Im \tau>0} \mathcal{C}\left(E_{\tau}\right)$, and $\mathcal{C}_{\text {triv }}=\cup_{\Im \tau>0} \mathcal{C}\left(E_{\tau}, \mathcal{O}_{E_{\tau}}\right)$, where $E_{\tau}=\mathbb{C} /(\mathbb{Z}+\mathbb{Z} \tau)$. For any of these moduli spaces, we can consider the Riemann-Hilbert correspondence map

$$
R H:\left(E_{\tau}, \mathcal{L}, \nabla\right) \longmapsto\left(\rho_{\nabla}(a), \rho_{\nabla}(b)\right),
$$

where $\rho_{\nabla}$ is the monodromy representation of $\nabla$, and $(a, b)$ is a basis of $\pi_{1}(E)$ corresponding to the basis $(1, \tau)$ of the period lattice $\mathbb{Z}+\mathbb{Z} \tau$. Remark that $\left.R H\right|_{\mathcal{C}(E, \mathcal{L})}$ cannot be surjective by dimensional reasons. The next proposition shows that $\left.R H\right|_{\mathcal{C}_{\text {triv }}}$ is dominant, though nonsurjective, and that $\left.R H\right|_{\mathcal{C}}$ is surjective.

Proposition 4. In the above notation,

$$
R H\left(\mathcal{C}_{\text {triv }}\right)=\left(\mathbb{C}^{*} \times \mathbb{C}^{*} \backslash\left\{S^{1} \times S^{1}\right\}\right) \cup\{(1,1)\}, \quad R H(\mathcal{C})=\mathbb{C}^{*} \times \mathbb{C}^{*} .
$$

Proof. Let $\nabla=\mathrm{d}+\omega$ be a connection on an elliptic curve $E$, where $\omega \in \Gamma\left(E_{\tau}, \Omega_{E_{\tau}}^{1}\right), A=\oint_{a} \omega$, $B=\oint_{b} \omega=\tau A$. By analytic continuation of solutions of the equation $\nabla \varphi=0$ along the cycles in $E$, we obtain $\rho(a)=e^{-A}$ and $\rho(b)=e^{-\tau A}$. The pair $(-A,-B)=(-A,-\tau A)$ is an element of $(0,0) \cup \mathbb{C}^{*} \times \mathbb{C}^{*}$. By setting $z=-A$, we deduce $R H\left(\mathcal{C}_{\text {triv }}\right)=\left\{\left(e^{z}, e^{z \tau}\right) \mid(z, \tau) \in \mathbb{C} \times \mathbb{H}\right\}$. The map exp : $\mathbb{C}^{*} \longrightarrow \mathbb{C}^{*}$ is surjective, so for all $w_{1} \in \mathbb{C}^{*}$, we can solve the equation $e^{z}=w_{1}$, and once we have fixed $z$, it is possible to solve $e^{\tau z}=w_{2}$ with respect to $\tau$ if and only if $\left(w_{1}, w_{2}\right) \notin S^{1} \times S^{1} \backslash\{(1,1)\}$. This ends the proof for $R H\left(\mathcal{C}_{\text {triv }}\right)$. The proof for $R H(\mathcal{C})$ is similar to the genus- 2 case, see Proposition 5 below.

From now on, we turn to the genus- 2 case. We define the moduli spaces $\mathcal{C}_{2}(C, \mathcal{L}), \mathcal{C}_{2}(C)$, $\mathcal{C}_{2}, \mathcal{C}_{2 \text {,triv }}$ similarly to the above, so that $\mathcal{C}_{2}(C)=\cup_{[\mathcal{L}] \in J(C)} \mathcal{C}_{2}(C, \mathcal{L}), \mathcal{C}_{2}=\cup_{Z \in \mathcal{H}} \mathcal{C}_{2}\left(C_{Z}\right)$, and $\mathcal{C}_{\text {triv }}=\cup_{Z \in \mathcal{H}} \mathcal{C}_{2}\left(C_{Z}, \mathcal{O}_{C_{Z}}\right)$. Here $\mathcal{H}$ is the locus of periods introduced in Corollary $1, C_{Z}$ is the genus-2 curve with period $Z, J_{2}(C)=\mathbb{C}^{2} / \Lambda$, where $\Lambda \simeq \mathbb{Z}^{4}$ is the lattice generated by the column vectors of the full period matrix $(1 \mid Z)$ of $C$. The Riemann-Hilbert correspondence is the map

$$
R H:\left(C_{Z}, \mathcal{L}, \nabla\right) \longmapsto\left(\rho_{\nabla}\left(\alpha_{1}\right), \rho_{\nabla}\left(\alpha_{2}\right), \rho_{\nabla}\left(\beta_{1}\right), \rho_{\nabla}\left(\beta_{2}\right)\right) \in\left(\mathbb{C}^{*}\right)^{4},
$$

where the generators $\alpha_{i}, \beta_{i}$ of $\pi_{1}(C)$ correspond to the basis of the lattice $\Lambda$.

Proposition 5. In the above notation,

$$
\begin{aligned}
& R H\left(\mathcal{C}_{2, \text { triv }}\right)=\left\{w \in\left(\mathbb{C}^{*}\right)^{4} \mid\left(w_{1} w_{2}, w_{3} w_{4}\right) \in W,\left(\frac{w_{1}}{w_{2}}, \frac{w_{3}}{w_{4}}\right) \in W\right\}, \\
& R H\left(\mathcal{C}_{2}\right)=\left(\mathbb{C}^{*}\right)^{4},
\end{aligned}
$$

where $W$ denotes the locus $R H\left(\mathcal{C}_{\text {triv }}\right)$ determined in Proposition 4. 
Proof. Let $\nabla=\mathrm{d}+\omega, \omega \in \Gamma\left(C_{Z}, \Omega_{C_{Z}}^{1}\right)$. We can consider $C_{Z}$ in its Abel-Jacobi embedding in $J C$, then $\omega=\lambda_{1} \mathrm{~d} z_{1}+\lambda_{2} \mathrm{~d} z_{2}$, where $\left(z_{1}, z_{2}\right)$ are the standard flat coordinates on $\mathbb{C}^{2} / \Lambda$. Therefore,

$$
R H\left(C_{Z}, \mathcal{O}_{Z}, \nabla\right)=\left(e^{\lambda_{1} z_{1}}, e^{\lambda_{2} z_{2}}, e^{\frac{1}{2}\left(\tau+\tau^{\prime}\right) \lambda_{1} z_{1}+\frac{1}{2}\left(\tau-\tau^{\prime}\right) \lambda_{2} z_{2}}, e^{\frac{1}{2}\left(\tau-\tau^{\prime}\right) \lambda_{1} z_{1}+\frac{1}{2}\left(\tau+\tau^{\prime}\right) \lambda_{2} z_{2}}\right) .
$$

Denoting the latter 4 -vector by $w$, we see that $\left(w_{1} w_{2}, w_{3} w_{4}\right)=\left(e^{z}, e^{\tau z}\right)$ with $z=\lambda_{1} z_{1}+\lambda_{2} z_{2}$, and $\left(\frac{w_{1}}{w_{2}}, \frac{w_{3}}{w_{4}}\right)=\left(e^{z^{\prime}}, e^{\tau^{\prime} z^{\prime}}\right)$ with $z^{\prime}=\lambda_{1} z_{1}-\lambda_{2} z_{2}$.

Then Proposition 4 implies the answer for $R H\left(\mathcal{C}_{2, \text { triv }}\right)$. Now, we will prove the surjectivity of $\left.R H\right|_{\mathcal{C}_{2}}$. On a genus-2 curve, any line bundle of degree 0 can be represented in the form $\mathcal{L}=\mathcal{O}_{C}\left(P_{1}+P_{2}-Q_{1}-Q_{2}\right)$ for some 4 points $P_{i}, Q_{i} \in C$. It is defined by its stalks: for any $P \in C, \mathcal{L}_{P}=\mathcal{O}_{P}$ if $P \notin\left\{P_{1}, P_{2}, Q_{1}, Q_{2}\right\}, \mathcal{L}_{P_{i}}=\frac{1}{\tau_{P_{i}}} \mathcal{O}_{P_{i}}, \mathcal{L}_{Q_{i}}=\tau_{Q_{i}} \mathcal{O}_{Q_{i}}$, where $\tau_{P}$ denotes a local parameter at $P$ for any $P \in C$. This implies that the constant function $e=1$ considered as a section of $\mathcal{L}$ has simple zeros at $P_{i}$ and simple poles at $Q_{i}$, that is, for its divisor we can write: $(e)=P_{1}+P_{2}-Q_{1}-Q_{2}$. According to [2], any line bundle of degree 0 admits a connection, and two connections differ by a holomorphic 1 -form. Hence any connection on $\mathcal{L}$ can be written in the form $\nabla=d+\omega, \omega=\nu+\lambda_{1} d z_{1}+\lambda_{2} d z_{2}$, where $\nu$ is a meromorphic 1-form with simple poles at $P_{i}, Q_{i}$ such that $\operatorname{Res}_{P_{i}} \nu=1, \operatorname{Res}_{Q_{i}} \nu=-1$ (these are apparent singularities of $\nabla$ with respect to the meromorphic trivialization $e=1$ ).

We can choose the coefficients $\lambda_{1}, \lambda_{2}$ in such a way that $\omega$ will have zero $a$-periods. Let us denote the periods of $\omega$ by $N_{i}$ :

$$
N_{1}=\int_{a_{1}} \omega, \quad N_{2}=\int_{a_{2}} \omega, \quad N_{3}=\int_{b_{1}} \omega, \quad N_{4}=\int_{b_{2}} \omega
$$

Then $N_{1}=N_{2}=0$ by the choice of $\omega$, and

$$
N_{2+j}=2 \pi i \sum_{k} \operatorname{Res}_{s_{k}}(\omega) \int_{s_{0}}^{s_{k}} d z_{j}, \quad j=1,2,
$$

by the Reciprocity Law for differentials of $1^{\text {st }}$ and $3^{\text {rd }}$ kinds [8, Section 2.2], where $\sum_{k} s_{k}$ is the divisor of poles $(\omega)_{\infty}$ of $\omega$, and $s_{0}$ is any point of $C$. Taking into account that $(\omega)_{\infty}=(\nu)_{\infty}=$ $P_{1}+P_{2}+Q_{1}+Q_{2}, \operatorname{Res}_{P_{i}} \nu=1, \operatorname{Res}_{Q_{i}} \nu=-1$, and $z_{j}(P)=\int_{P_{0}}^{P} d z_{j}$, we can rewrite:

$$
N_{2+j}=2 \pi i\left[z_{j}\left(P_{1}\right)-z_{j}\left(Q_{1}\right)+z_{j}\left(P_{2}\right)-z_{j}\left(Q_{2}\right)\right] .
$$

Hence the components of the vector $\frac{1}{2 \pi i}\left(\begin{array}{c}N_{3} \\ N_{4}\end{array}\right)$ are the 2 coordinates on $J C$ of the class $[\mathcal{L}]$ of the line bundle $\mathcal{L}$, which is the same as the divisor class $\left[P_{1}+P_{2}-Q_{1}-Q_{2}\right]$. Now, we can finish the proof.

Let $\left(w_{i}\right) \in\left(\mathbb{C}^{*}\right)^{4}$. Then, we can find a 1 -form $\eta_{1}$ of a connection on a degree-0 line bundle $\mathcal{L}_{1}$ with monodromy $\left(1,1, w_{3}, w_{4}\right)$ in choosing $\mathcal{L}_{1}$ with coordinates $-\frac{1}{2 \pi i}\left(\log w_{3}, \log w_{4}\right)$ on $J C$. In interchanging the roles of $a$ - and $b$-periods, we will find another 1 -form of connection $\eta_{2}$ on another degree- 0 line bundle $\mathcal{L}_{2}$, with monodomy $\left(w_{1}, w_{2}, 1,1\right)$. Then $\omega=\eta_{1}+\eta_{2}$ is the form of a connection on $\mathcal{L}_{1} \otimes \mathcal{L}_{2}$ with monodromy $\left(w_{i}\right) \in\left(\mathbb{C}^{*}\right)^{4}$.

\section{Direct images of rank-1 connections}

We will determine the direct image connections $f_{*}\left(\nabla_{\mathcal{L}}\right)=\nabla_{\mathcal{E}}$ on the rank-2 vector bundle $\mathcal{E}=f_{*} \mathcal{L}$, where $f: C \rightarrow E$ is an elliptic subcover of degree 2 of $C$. From now on, we will stick to a representation of $C$ in the classical form $y^{2}=F_{6}(\xi)$, where $F_{6}$ is a degree-6 polynomial. We 
want that $E$ is given the Legendre equation $y^{2}=x(x-1)(x-t)$, but $F_{6}$ is not so complicated as in (5). Of course, this can be done in many different ways. We will fix for $C$ and $f$ the following choices:

$$
\begin{aligned}
& \left.f: C=\left\{y^{2}=\left(t^{\prime}-\xi^{2}\right)\left(t^{\prime}-1-\xi^{2}\right)\left(t^{\prime}-t-\xi^{2}\right)\right\} \rightarrow E=\left\{y^{2}=x(x-1)(x-t)\right)\right\}, \\
& (\xi, y) \mapsto(x, y)=\left(t^{\prime}-\xi^{2}, y\right) .
\end{aligned}
$$

Lemma 2. For any bielliptic curve $C$ with an elliptic subcover $f: C \rightarrow E$ of degree 2 , there exist affine coordinates $\xi, x, y$ on $C, E$ such that $f, C, E$ are given by (7) for some $t, t^{\prime} \in \mathbb{C} \backslash\{0,1\}$, $t \neq t^{\prime}$

Proof. By Proposition 1, it suffices to verify that the two elliptic subcovers $E, E^{\prime}$ of the curves $C$ given by (7), as we vary $t, t^{\prime}$, run over the whole moduli space of elliptic curves independently from each other. $E^{\prime}$ can be determined from (2). It is a double cover of $\mathbb{P}^{1}$ ramified at $\frac{1}{t^{\prime}}, \frac{1}{t^{\prime}-1}, \frac{1}{t^{\prime}-t}, \infty$. This quadruple can be sent by a homographic transformation to $0,1, t, t^{\prime}$, hence $E^{\prime}$ is given by $y^{2}=x(x-1)(x-t)\left(x-t^{\prime}\right)$. If we fix $t$ and let vary $t^{\prime}$, we will obviously obtain all the elliptic curves, which ends the proof.

The only branch points of $f$ in $E$ are $p_{ \pm}=\left(t^{\prime}, \pm y_{0}\right)$, where $y_{0}=\sqrt{t^{\prime}\left(t^{\prime}-1\right)\left(t^{\prime}-t\right)}$, and thus the ramification points of $f$ in $C$ are $\tilde{p}_{ \pm}=\left(0, \pm y_{0}\right)$. In particular, $f$ is non-ramified at infinity and the preimage of $\infty \in E$ is a pair of points $\infty_{ \pm} \in C$. E is the quotient of $C$ by the involution $\iota: C \rightarrow C$, called the Galois involution of the double covering $f$. It is given in coordinates by $\iota:(\xi, y) \mapsto(-\xi, y)$.

We first deal with the case when $\mathcal{L}$ is the trivial bundle $\mathcal{O}_{C}$, in which we write $\nabla_{\mathcal{O}}$ instead of $\nabla_{\mathcal{L}}$. The direct image $\mathcal{E}_{0}=f_{*} \mathcal{O}_{C}$ is a vector bundle of rank 2 which splits into the direct sum of the $\iota$-invariant and anti-invariant subbundles: $\mathcal{E}_{0}=\left(f_{*} \mathcal{O}_{C}\right)^{+} \oplus\left(f_{*} \mathcal{O}_{C}\right)^{-}$. The latter subbundles are defined as sheaves by specifying their sections over any open subset $U$ of $E$ :

$$
\Gamma\left(U,\left(f_{*} \mathcal{O}_{C}\right)^{ \pm}\right)=\left\{s \in \Gamma\left(f^{-1}(U), \mathcal{O}_{C}\right) \mid \iota^{*}(s)= \pm s\right\} .
$$

Obviously, the $\iota$-invariant sections are just functions on $E$, so the first direct summand $\left(f_{*} \mathcal{O}_{C}\right)^{+}$ is the trivial bundle $\mathcal{O}_{E}$. The second one is generated over the affine set $E \backslash\{\infty\}$ by a single generator $\xi$, one of the two coordinates on $C$. Thus, we can use $(1, \xi)$ as a basis trivializing $\mathcal{E}_{0}$ over $E \backslash\{\infty\}$ and compute $\nabla=f_{*}\left(\nabla_{\mathcal{O}}\right)$ in this basis. We use, of course, the constant function 1 to trivialize $\mathcal{O}_{C}$ and write $\nabla_{\mathcal{O}}$ in the form

$$
\nabla_{\mathcal{O}}=d+\omega, \quad \omega=\nabla_{\mathcal{O}}(1)=\lambda_{1} \frac{\mathrm{d} \xi}{y}+\lambda_{2} \frac{\xi \mathrm{d} \xi}{y} .
$$

Re-writing $\nabla_{\mathcal{O}}(1)=\omega$ in terms of the coordinate $x=t^{\prime}-\xi^{2}$, we get:

$$
\nabla_{\mathcal{O}}(1)=-\frac{\lambda_{1}}{2\left(t^{\prime}-x\right)} \frac{\mathrm{d} x}{y} \xi-\frac{\lambda_{2}}{2} \frac{\mathrm{d} x}{y} 1 .
$$

Likewise,

$$
\nabla_{\mathcal{O}}(\xi)=-\frac{\lambda_{1}}{2} \frac{\mathrm{d} x}{y} 1-\frac{\lambda_{2}}{2 y} \frac{\mathrm{d} x}{y} \xi-\frac{\mathrm{d} x}{2\left(t^{\prime}-x\right)} \xi .
$$

We obtain the matrix of $\nabla=f_{*}\left(\nabla_{\mathcal{O}}\right)$ in the basis $(1, \xi)$ :

$$
A=\left(\begin{array}{cc}
-\frac{\lambda_{2}}{2 y} \mathrm{~d} x & -\frac{\lambda_{1}}{2 y} \mathrm{~d} x \\
-\frac{\lambda_{1}}{2\left(t^{\prime}-x\right) y} \mathrm{~d} x & -\left(\frac{\lambda_{2}}{2 y}+\frac{1}{2\left(t^{\prime}-x\right)}\right) \mathrm{d} x
\end{array}\right) .
$$


This matrix has poles at the branch points $p_{ \pm}$with residues

$$
\operatorname{Res}_{p_{+}} A=\left(\begin{array}{cc}
0 & 0 \\
\frac{\lambda_{1}}{2 y_{0}} & \frac{1}{2}
\end{array}\right), \quad \operatorname{Res}_{p_{-}} A=\left(\begin{array}{cc}
0 & 0 \\
-\frac{\lambda_{1}}{2 y_{0}} & \frac{1}{2}
\end{array}\right) .
$$

As the sum of residues of a meromorphic 1-form on a compact Riemann surface is zero, we can evaluate the residue at infinity:

$$
\operatorname{Res}_{p_{-}} A+\operatorname{Res}_{p_{+}} A=-\operatorname{Res}_{\infty}(A)=\left(\begin{array}{cc}
0 & 0 \\
0 & 1
\end{array}\right)
$$

It is nonzero, hence $A$ is not regular at $\infty$ and has exactly 3 poles on $E$. In fact, the pole at $\infty$ is an apparent singularity due to the fact that $(1, \xi)$ fails to be a basis of $f_{*} \mathcal{O}_{C}$ at $\infty$, which follows from the following proposition:

Proposition 6. Let $f: C \rightarrow E$ be the bielliptic cover (7), and $\nabla_{\mathcal{O}}=d+\omega$ a regular connection on the trivial bundle $\mathcal{O}_{C}$ with connection form $\omega=\lambda_{1} \frac{\mathrm{d} \xi}{y}+\lambda_{2} \frac{\xi \mathrm{d} \xi}{y}$. Then the direct image $\nabla=f_{*}\left(\nabla_{\mathcal{O}}\right)$ is a logarithmic connection on a rank-2 vector bundle $\mathcal{E}_{0}$ over $E$, whose only poles are the two branch points $p_{ \pm}$of $f$. In an appropriate trivialization of $\mathcal{E}_{0}$ over $E \backslash\{\infty\}, \nabla$ is given by the connection matrix (9), and the residues at $p_{ \pm}$are given by (10).

Proof. If $P \in E$ is not a branch point, then we can choose a small disk $U$ centered at $P$ such that $f^{-1}(U)$ is the disjoint union of two disks $U_{ \pm}$. Let $e_{ \pm}$be a nonzero $\nabla_{\mathcal{O}}$-flat section of $\mathcal{O}_{C}$ over $U_{ \pm}$. Then $\left(e_{+}, e_{-}\right)$is a basis of $\mathcal{E}_{0}$ over $U$ consisting of $\nabla$-flat sections. This implies the regularity of $\nabla$ over $U$ (the connection matrix of $\nabla$ in this basis is zero).

We have shown that the only points where the direct image of a regular connection might have singularities are the branch points of the covering. In particular, $\infty$ is not a singularity of $\nabla$. The fact that the branch points are logarithmic poles follows from the calculation preceding the statement of the proposition.

At this point, it is appropriate to comment on the horizontal sections of $\nabla$, which are solutions of the matrix ODE $d \Phi+A \Phi=0$ for the vector $\Phi=\left(\begin{array}{c}\Phi_{1} \\ \Phi_{2}\end{array}\right)$. We remark that the matrix ODE is equivalent to one scalar equation of second order which we have not encountered in the literature. It is obtained as follows: the first line of the matrix equation gives

$$
\Phi_{2}=\frac{2 \lambda_{2}}{\lambda_{1}} \Phi_{1}^{\prime}-\frac{\lambda_{2}}{\lambda_{1}} \Phi_{1}
$$

where $\Phi_{1}, \Phi_{2}$ denote the components of a single 2 -vector $\Phi$, and the prime denotes the derivative with respect to $x$. The second equation gives:

$$
\Phi_{2}^{\prime}=\frac{\lambda_{1}}{2 y\left(t^{\prime}-x\right)} \Phi_{1}+\left(\frac{\lambda_{2}}{2 y}+\frac{1}{2\left(t^{\prime}-x\right)}\right) \Phi_{2} .
$$

By substituting here $\Phi_{2}$ in terms of $\Phi_{1}$, we get one second order equation for $\Phi_{1}$. By setting $y^{2}=P_{3}(x)=x(x-1)(x-t)$, we have $y^{\prime}=\frac{P_{3}^{\prime}}{2 y}$ and the differential equation for $\Phi_{1}$ takes the form

$$
\Phi_{1}^{\prime \prime}+\left[\frac{P_{3}^{\prime}(x)}{2 P_{3}(x)}-\frac{\lambda_{2}}{y}+\frac{1}{2\left(x-t^{\prime}\right)}\right] \Phi_{1}^{\prime}+\left[\frac{\lambda_{1}^{2}}{4 P_{3}(x)\left(x-t^{\prime}\right)}+\frac{\lambda_{2}^{2}}{4 P_{3}(x)}-\frac{\lambda_{2}}{4\left(x-t^{\prime}\right) y}\right] \Phi_{1}=0 .
$$

We can also write out the second order differential equation for $\Phi_{1}$ with respect to the flat coordinate $z=\int \frac{\mathrm{d} x}{y}$ on $E$. Now, set up the convention that the prime denotes $\frac{\mathrm{d}}{\mathrm{d} z}$. Then, after 
an appropriate scaling, $x=\wp(z)+\frac{t+1}{3}, y=\frac{\wp^{\prime}(z)}{2}$. Let $z_{0},-z_{0}$ be the solutions of $\wp(z)=t^{\prime}-\frac{t+1}{3}$ modulo the lattice of periods. Then we have the following equation for $\Phi_{1}$ :

$$
\Phi_{1}^{\prime \prime}+\left[-\lambda_{2}+\frac{\wp^{\prime}(z)}{2\left(\wp(z)-\wp\left(z_{0}\right)\right)}\right] \Phi_{1}^{\prime}+\left[\frac{\lambda_{2}^{2}}{4}+\frac{2 \lambda_{1}^{2}-\lambda_{2} \wp^{\prime}(z)}{8\left(\wp(z)-\wp\left(z_{0}\right)\right)}\right] \Phi_{1}=0 .
$$

We now go over to the general case, in which $\mathcal{L}$ is any line bundle of degree 0 on $C$ endowed with a regular connection $\nabla_{\mathcal{L}}$. Then $f_{*} \mathcal{L}=\mathcal{E}$ is a vector bundle of rank 2 on $E$ endowed with a logarithmic connection $\nabla_{\mathcal{E}}=f_{*} \nabla_{\mathcal{L}}$. We can represent $\mathcal{L}$ in the form $\mathcal{L}=\mathcal{O}\left(\tilde{q}_{1}+\tilde{q}_{2}-\infty_{+}-\infty_{-}\right)$ with $\tilde{q}_{1}=\left(\xi_{1}, y_{1}\right)$ and $\tilde{q}_{2}=\left(\xi_{2}, y_{2}\right)$ some points of $C$. Their images on $E$ will be denoted by $q_{i}$, or $\left(x_{i}, y_{i}\right)$ in coordinates. We will use $1 \in \Gamma\left(\mathcal{O}_{C}\right)$ as a meromorphic trivialization of $\mathcal{L}$ as in the proof of Proposition 5. In this trivialization, the connection form of $\nabla_{\mathcal{L}}$ has simple poles at the 4 points $\tilde{q}_{i}, \infty_{ \pm}$with residues +1 at $\tilde{q}_{i}$ and -1 at $\infty_{ \pm}$. It is easy to invent one example of such a form: $\nu=\frac{1}{2}\left(\frac{y+y_{1}}{\xi-\xi_{1}}+\frac{y+y_{2}}{\xi-\xi_{2}}\right) \frac{\mathrm{d} \xi}{y}$. Hence the general form of $\nabla_{\mathcal{L}}$ is as follows:

$$
\nabla_{\mathcal{L}}=\mathrm{d}+\omega=\mathrm{d}+\frac{1}{2}\left(\frac{y+y_{1}}{\xi-\xi_{1}}+\frac{y+y_{2}}{\xi-\xi_{2}}\right) \frac{\mathrm{d} \xi}{y}+\lambda_{1} \frac{\mathrm{d} \xi}{y}+\lambda_{2} \frac{\xi \mathrm{d} \xi}{y}
$$

We compute $\nabla_{\mathcal{L}}(1)$ and $\nabla_{\mathcal{L}}(\xi)$ and express the result in the coordinates $(x, y)$ of $E$. This brings us to formulas for the connection $\nabla_{\mathcal{E}}$ on $E$. We obtain:

$$
\nabla_{\mathcal{L}}(1)=\omega \cdot 1=\left[\frac{1}{2}\left(\frac{y+y_{1}}{\xi\left(\xi-\xi_{1}\right)}+\frac{y+y_{2}}{\xi\left(\xi-\xi_{2}\right)}\right)+\frac{\lambda_{1}}{\xi}+\lambda_{2}\right] \frac{\xi \mathrm{d} \xi}{y} .
$$

Splitting $\frac{1}{\xi-\xi_{i}}$ into the invariant and anti-invariant parts, we get:

$$
\begin{aligned}
\nabla_{\mathcal{L}}(\xi)= & \xi \nabla_{\mathcal{L}}(1)+\mathrm{d} \xi \cdot 1=\left[\frac{1}{2}\left(\frac{\left(y+y_{1}\right) \xi_{1}}{\xi^{2}-\xi_{1}^{2}}+\frac{\left(y+y_{2}\right) \xi_{2}}{\xi^{2}-\xi_{2}^{2}}\right)+\lambda_{1}\right. \\
& \left.+\frac{1}{2}\left(\frac{y+y_{1}}{\xi^{2}-\xi_{1}^{2}}+\frac{y+y_{2}}{\xi^{2}-\xi_{2}^{2}}\right) \xi+\lambda_{2} \xi+\frac{y}{\xi^{2}} \xi\right] \frac{\xi \mathrm{d} \xi}{y}
\end{aligned}
$$

By using the relations $x=t^{\prime}-\xi^{2}, \mathrm{~d} x=-2 \xi \mathrm{d} \xi$, we determine the connection $\nabla_{\mathcal{E}}=d+A$, where $A$ is the matrix of $\nabla_{\mathcal{E}}$ in the basis $(1, \xi)$ :

$$
\left(\begin{array}{cc}
-\frac{1}{2}\left(\frac{1}{2}\left(\frac{y+y_{1}}{x_{1}-x}+\frac{y+y_{2}}{x_{2}-x}\right)+\lambda_{2}\right) \frac{\mathrm{d} x}{y} & -\frac{1}{2}\left(\frac{1}{2}\left(\frac{\left(y+y_{1}\right) \xi_{1}}{x_{1}-x}+\frac{\left(y+y_{2}\right) \xi_{2}}{x_{2}-x}\right)+\lambda_{1}\right) \frac{\mathrm{d} x}{y} \\
-\frac{1}{2}\left(\frac{1}{2}\left(\frac{\left(y+y_{1}\right) \xi_{1}}{\left(x_{1}-x\right)\left(t^{\prime}-x\right)}+\frac{\left(y+y_{2}\right) \xi_{2}}{\left(x_{2}-x\right)\left(t^{\prime}-x\right)}\right)+\frac{\lambda_{1}}{t^{\prime}-x}\right) \frac{\mathrm{d} x}{y} & -\frac{1}{2}\left(\frac{1}{2}\left(\frac{y+y_{1}}{x_{1}-x}+\frac{y+y_{2}}{x_{2}-x}\right)+\lambda_{2}+\frac{y}{t^{\prime}-x}\right) \frac{\mathrm{d} x}{y}
\end{array}\right) .
$$

We compute $\operatorname{Res}_{p_{ \pm}} A$, where $p_{ \pm}$are the only singularities of $\nabla_{\mathcal{E}}$ :

$$
\operatorname{Res}_{p_{ \pm}} A=\left(\begin{array}{cc}
0 & 0 \\
\frac{1}{4}\left(\frac{\left(y_{1} \pm y_{0}\right) \xi_{1}}{x_{1}-t^{\prime}}+\frac{\left(y_{2} \pm y_{0}\right) \xi_{2}}{x_{2}-t^{\prime}}\right) \pm \frac{\lambda_{1}}{2 y_{0}} & \frac{1}{2}
\end{array}\right) .
$$

Proposition 7. Let $f: C \rightarrow E$ be the bielliptic cover $(7), \mathcal{L}=\mathcal{O}\left(\tilde{q}_{1}+\tilde{q}_{2}-\infty_{+}-\infty_{-}\right)$with $\tilde{q}_{i}=\left(\xi_{i}, y_{i}\right) \in C(i=1,2)$, and $\nabla_{\mathcal{L}}=d+\omega$ a regular connection on $\mathcal{L}$ with connection form $\omega$ defined by (11). Assume that $\xi_{i} \neq 0$, that is $\tilde{q}_{i} \neq \tilde{p}_{ \pm}$. Then the direct image $\nabla_{\mathcal{E}}=f_{*}\left(\nabla_{\mathcal{L}}\right)$ is a logarithmic connection on a rank-2 vector bundle $\mathcal{E}$ over $E$ whose only poles are the two branch points $p_{ \pm}$of $f$. In the meromorphic trivialization of $\mathcal{E}$ defined by $(1, \xi), \nabla_{\mathcal{E}}$ is given by the connection matrix (12), and the residues at $p_{ \pm}$are given by (13). 

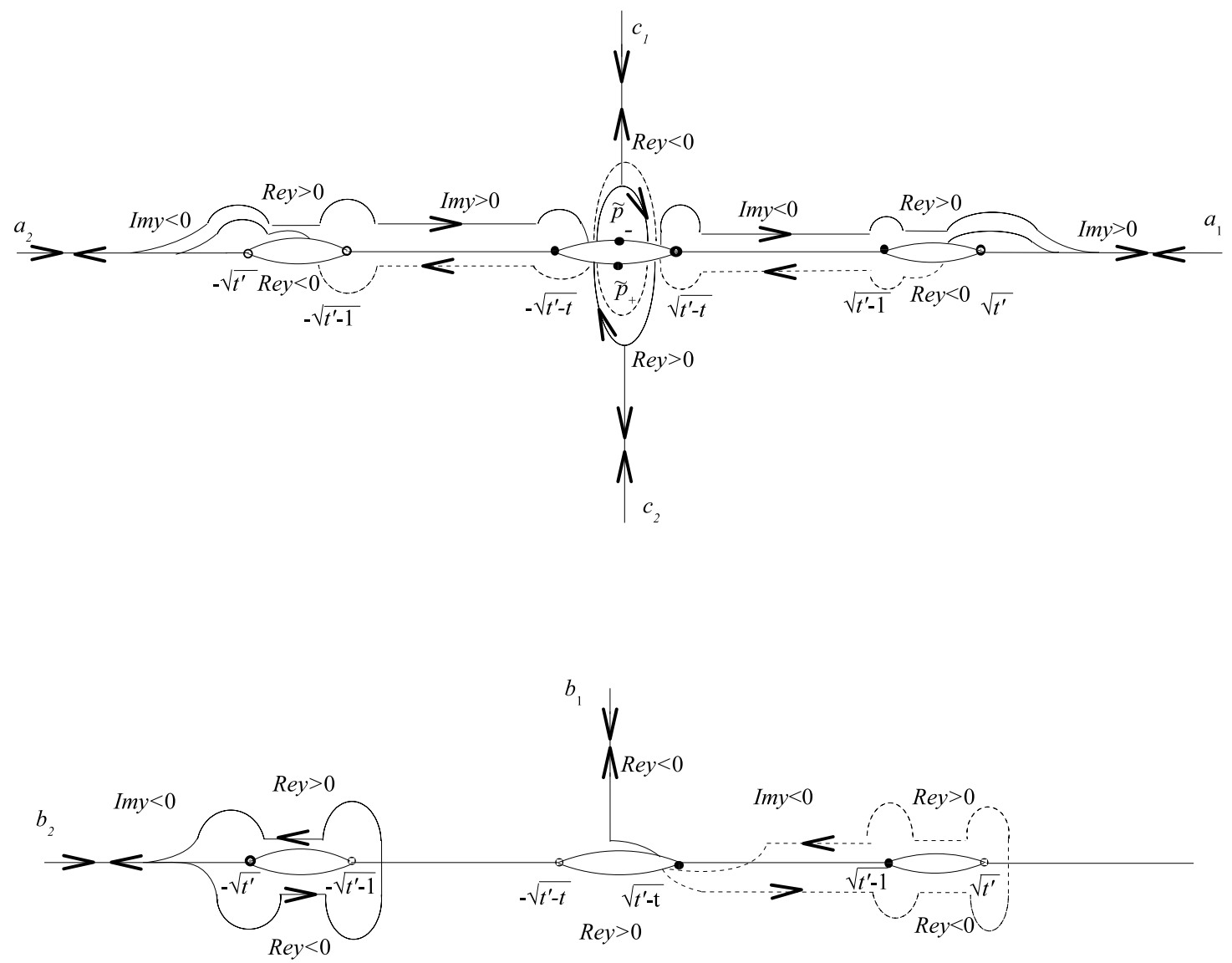

Figure 2. Generators of $\pi_{1}\left(C \backslash\left\{\tilde{p}_{ \pm}\right\}, \infty_{+}\right)$. The parts of the arcs represented in solid (resp. dash) lines are on the upper (resp. lower) sheet.

Remark that the points $q_{i}=f\left(\tilde{q}_{i}\right)=\left(x_{i}, y_{i}\right)$ are apparent singularities of $\nabla_{\mathcal{E}}$. We write down the residues of $A$ at these points for future use:

$$
\operatorname{Res}_{q_{1}} A=\left(\begin{array}{cc}
\frac{1}{2} & \frac{\xi_{1}}{2} \\
\frac{1}{2 \xi_{1}} & \frac{1}{2}
\end{array}\right), \quad \operatorname{Res}_{q_{2}} A=\left(\begin{array}{cc}
\frac{1}{2} & \frac{\xi_{2}}{2} \\
\frac{1}{2 \xi_{2}} & \frac{1}{2}
\end{array}\right) .
$$

We can also compute $\operatorname{Res}_{\infty} A$. First homogenize the equation of $E$ via the change $x=\frac{x_{1}}{x_{0}}$, and $y=\frac{x_{2}}{x_{0}}$. The homogeneous equation is $x_{0} x_{2}^{2}=x_{1}^{3}-(1+t) x_{1}^{2} x_{0}+t x_{1} x_{0}^{2}$. Then, setting $v=\frac{x_{0}}{x_{2}}, u=\frac{x_{1}}{x_{2}}$, we obtain the equation $v=u^{3}-(1+t) u^{2} v+t u v^{2}$ in the neighborhood of $\infty$. Near $\infty=(0,0)$, we have $v \sim u^{3}, \frac{\mathrm{d} x}{y} \sim-2 \mathrm{~d} u$. Therefore, $\operatorname{Res}_{\infty} A$ is:

$$
\operatorname{Res}_{\infty} A=\operatorname{Res}_{u=0} A=\left(\begin{array}{cc}
-1 & -\frac{\xi_{1}+\xi_{2}}{2} \\
0 & -2
\end{array}\right) .
$$

\section{Monodromy of direct image connections}

We are using the notation of the previous section. We will calculate the monodromy of the direct image connections $\nabla_{\mathcal{E}}$. We will start by choosing generators of the fundamental group $\pi_{1}\left(E \backslash\left\{p_{+}, p_{-}\right\}\right)$. To express the monodromy of $\nabla$ in terms of periods of $C$, we will first introduce generators $a_{i}, b_{i}, c_{i}$ for $\pi_{1}\left(C \backslash\left\{\tilde{p}_{+}, \tilde{p}_{-}\right\}\right)$, and then descend some of them to $E$ by applying $f_{*}$. We choose $\infty_{+}($resp. $\infty)$ as the reference point on $C$ (resp. E). For this definition, assume 
that $t, t^{\prime}$ are real and $1<t<t^{\prime}$ (for general $t, t^{\prime}$, the loops $a_{i}, b_{i}, c_{i}$ are defined up to an isotopy bringing $t, t^{\prime}$ onto the real axis so that $\left.1<t<t^{\prime}\right)$. $C$ can be represented as the result of gluing two copies of the Riemann sphere along three cuts. We call these copies of the Riemann sphere upper and lower sheets, and the cuts are realized along the rectilinear segments $\left[-\sqrt{t^{\prime}}\right.$, $\left.-\sqrt{t^{\prime}-1}\right],\left[-\sqrt{t^{\prime}-t}, \sqrt{t^{\prime}-t}\right]$ and $\left[\sqrt{t^{\prime}-1}, \sqrt{t^{\prime}}\right]$. The sheets are glued together in such a way that the upper edge of each cut on the upper sheet is identified with the lower edge of the respective cut on the lower sheet, and vice versa. Let $\infty_{+}$be on the upper sheet, singled out by the condition $\Im y>0$ when $\xi \in \mathbb{R}, \xi \rightarrow+\infty$. This implies that the values of $\Re y, \Im y$ on $\mathbb{R}$ are as on Fig. 2, where the loops $a_{i}, b_{i}, c_{i}$ generating $\pi_{1}\left(C \backslash\left\{\tilde{p}_{+}, \tilde{p}_{-}\right\}\right)$are shown. Remark that the loops $c_{i}$ are chosen in the form $c_{i}=d_{i} \tilde{c}_{i} d_{i}^{-1}$, where $d_{i}$ is a path joining $\infty_{+}$with some point close to $\tilde{p}_{ \pm}$and $\tilde{c}_{i}$ is a small circle around $\tilde{p}_{ \pm}$(the values $i=1,2$ correspond to $\tilde{p}_{+}, \tilde{p}_{-}$respectively). The paths $d_{i}$ follow the imaginary axis of the upper sheet.

Now, we go over to $E$. Set $a=f_{*}\left(a_{1}\right), b=f_{*}\left(b_{1}\right)$, and define the closed paths running round the branch points $p_{ \pm}$as follows: $\gamma_{i}=f\left(d_{i}\right) \tilde{\gamma}_{i} f\left(d_{i}\right)^{-1}$, where $\tilde{\gamma}_{i}$ are small circles around $p_{ \pm}$ running in the same direction as $f\left(\tilde{c}_{i}\right)$ (but $f\left(\tilde{c}_{i}\right)$ makes two revolutions around $p_{ \pm}$, whilst $\tilde{\gamma}_{i}$ only one).

One can verify that the thus defined generators of both fundamental groups satisfy the relations $\left[a_{1}, b_{1}\right] c_{1}\left[a_{2}, b_{2}\right] c_{2}=1$ and $[a, b] \gamma_{1} \gamma_{2}=1$ and that the group morphism $f_{*}: \pi_{1}\left(C \backslash\left\{\tilde{p}_{ \pm}\right\}, \infty_{+}\right) \longrightarrow \pi_{1}\left(E \backslash\left\{p_{ \pm}\right\}, \infty\right)$ is given by the formulas

$$
f_{*}\left(a_{1}\right)=a, \quad f_{*}\left(b_{1}\right)=b, \quad f_{*}\left(a_{2}\right)=\gamma_{1}^{-1} a \gamma_{1}, \quad f_{*}\left(b_{2}\right)=\gamma_{1}^{-1} b \gamma_{1}, \quad f_{*}\left(c_{i}\right)=\gamma_{i}^{2} \quad(i=1,2) .
$$

As $\nabla_{\mathcal{L}}$ is regular at $\tilde{p}_{ \pm}$, it has no monodromy along $c_{i}$, and this together with the above formulas for $f_{*}$ immediately implies that the monodromy matrices $M_{\gamma_{i}}$ of $\nabla_{\mathcal{E}}$ are of order 2.

We first assume that $\mathcal{L}=\mathcal{O}_{C}$ is trivial, in which case $\nabla_{\mathcal{L}}$ is denoted $\nabla_{\mathcal{O}}$, and $\nabla_{\mathcal{E}}$ just $\nabla$. As in the previous section, we trivialize $\mathcal{E}_{0}=f_{*}\left(\mathcal{O}_{C}\right)$ by the basis $(1, \xi)$ over $E \backslash\{\infty\}$. Splitting the solution $\varphi=e^{-\lambda_{1} z_{1}-\lambda_{2} z_{2}}$ of $\nabla_{\mathcal{O}} \varphi=0$ into the $\iota$-invariant and anti-invariant parts, we represent $\varphi$ by a 2 -component vector in the basis $(1, \xi)$ :

$$
\Phi=\left(\begin{array}{c}
e^{-\lambda_{2} z_{2}} \cosh \left(\lambda_{1} z_{1}\right) \\
-\frac{e^{-\lambda_{2} z_{2}}}{\xi} \sinh \left(\lambda_{1} z_{1}\right)
\end{array}\right) .
$$

We have to complete $\Phi$ to a fundamental matrix $\boldsymbol{\Phi}$, and then we can define the monodromy $M_{\gamma}$ along a loop $\gamma$ by $T_{\gamma}(\boldsymbol{\Phi})=\boldsymbol{\Phi} M_{\gamma}$, where $T_{\gamma}$ denotes the analytic continuation along $\gamma$. We already know the first column of $\boldsymbol{\Phi}$ : this is just $\Phi$. Denote it also by $\boldsymbol{\Phi}_{\mathbf{1}}$, the column vector $\left(\begin{array}{l}\Phi_{1,1} \\ \Phi_{2,1}\end{array}\right)$. It remains to find $\boldsymbol{\Phi}_{\mathbf{2}}=\left(\begin{array}{c}\Phi_{1,2} \\ \Phi_{2,2}\end{array}\right)$ so that

$$
\boldsymbol{\Phi}=\left(\begin{array}{ll}
\Phi_{1,1} & \Phi_{1,2} \\
\Phi_{2,1} & \Phi_{2,2}
\end{array}\right)
$$

is a fundamental matrix. By Liouville's theorem, the matrix equation $\boldsymbol{\Phi}^{\prime}+A \boldsymbol{\Phi}=0$ implies the following scalar equation for $\Psi=\operatorname{det} \Phi: \Psi^{\prime}+\operatorname{Tr}(A) \Psi=0$. In our case, $\operatorname{Tr}(A)=-\frac{\lambda_{2}}{y}-\frac{1}{2\left(t^{\prime}-x\right)}$, and we get a solution in the form: $\Psi=\frac{e^{-2 \lambda_{2} z_{2}}}{\sqrt{t^{\prime}-x}}=\frac{e^{-2 \lambda_{2} z_{2}}}{\xi}$. Thus we can determine $\boldsymbol{\Phi}_{2}$ from the system:

$$
\begin{aligned}
& \cosh \left(\lambda_{1} z_{1}\right) \Phi_{2,2}+\frac{1}{\xi} \sinh \left(\lambda_{1} z_{1}\right) \Phi_{1,2}=\frac{e^{-2 \lambda_{2} z_{2}}}{\xi}, \\
& \Phi_{1,2}^{\prime}=\frac{1}{2 y}\left(\lambda_{2} \Phi_{1,2}+\lambda_{1} \Phi_{2,2}\right) .
\end{aligned}
$$


Eliminating $\Phi_{2,2}$, we obtain an inhomogeneous first order linear differential equation for $\Phi_{1,2}$. Finally, we find:

$$
\mathbf{\Phi}_{\mathbf{2}}=\left(\begin{array}{c}
-e^{-\lambda_{2} z_{2}} \sinh \left(\lambda_{1} z_{1}\right) \\
\frac{e^{-\lambda_{2} z_{2}}}{\xi} \cosh \left(\lambda_{1} z_{1}\right)
\end{array}\right) .
$$

Now we can compute the monodromies of $\nabla_{\mathcal{E}}$ along the loops $a, b, \gamma_{i}$. It is convenient to represent the result in a form, in which the real and imaginary parts of all the entries are visible as soon as $t, t^{\prime} \in \mathbb{R}$ and $1<t<t^{\prime}$. Under this assumption, the entries of the period matrix $\Pi=\left(\left(a_{i j} \mid\left(b_{i j}\right)\right.\right.$ of $C$ are real or imaginary and can be expressed in terms of hyperelliptic integrals along the real segments joining branch points.

Thus reading the cycles of integration from Fig. 2, we obtain:

$$
\begin{array}{ll}
a_{1,1}=-a_{1,2}=2 i K, \quad K=\int_{\sqrt{t^{\prime}-t}}^{\sqrt{t^{\prime}-1}} \frac{\mathrm{d} \xi}{\sqrt{\left(t^{\prime}-\xi^{2}\right)\left(t^{\prime}-1-\xi^{2}\right)\left(t-t^{\prime}+\xi^{2}\right)}}>0, \\
a_{2,1}=a_{2,2}=2 i K^{\prime}, \quad K^{\prime}=\int_{\sqrt{t^{\prime}-t}}^{\sqrt{t^{\prime}-1}} \frac{\xi \mathrm{d} \xi}{\sqrt{\left(t^{\prime}-\xi^{2}\right)\left(t^{\prime}-1-\xi^{2}\right)\left(t-t^{\prime}+\xi^{2}\right)}}>0, \\
b_{1,1}=-b_{1,2}=-2 L, & L=\int_{\sqrt{t^{\prime}}}^{\sqrt{t^{\prime}-1}} \frac{\mathrm{d} \xi}{|y|}>0, \\
b_{2,1}=b_{2,2}=-2 L^{\prime}, & L^{\prime}=\int_{\sqrt{t^{\prime}-1}}^{\sqrt{t^{\prime}}} \frac{\xi \mathrm{d} \xi}{|y|}>0 .
\end{array}
$$

Proposition 8. The monodromy matrices of the connection $\nabla=f_{*}\left(\nabla_{\mathcal{O}}\right)$, where $\nabla_{\mathcal{O}}$ is the rank-1 connection (8), are given by

$$
\begin{aligned}
& M_{a}=\left(\begin{array}{cc}
e^{-2 i \lambda_{2} K^{\prime}} \cos \left(2 \lambda_{1} K\right) & -e^{-2 i \lambda_{2} K^{\prime}} i \sin \left(2 \lambda_{1} K\right) \\
-e^{-2 i \lambda_{2} K^{\prime}} i \sin \left(2 \lambda_{1} K\right) & e^{-2 i \lambda_{2} K^{\prime}} \cos \left(2 \lambda_{1} K\right)
\end{array}\right), \\
& M_{b}=\left(\begin{array}{cc}
e^{2 \lambda_{2} L^{\prime}} \cosh \left(2 \lambda_{1} L\right) & e^{2 \lambda_{2} L^{\prime}} \sinh \left(2 \lambda_{1} L\right) \\
e^{2 \lambda_{2} L^{\prime}} \sinh \left(2 \lambda_{1} L\right) & e^{2 \lambda_{2} L^{\prime}} \cosh \left(2 \lambda_{1} L\right)
\end{array}\right), \\
& M_{\gamma_{i}}=\left(\begin{array}{cc}
1 & 0 \\
0 & -1
\end{array}\right) \quad(i=1,2) .
\end{aligned}
$$

Now we turn to the general case of nontrivial $\mathcal{L}$. Our computations done in the special case allow us to guess the form of the fundamental matrix of solutions to $\nabla_{\mathcal{E}} \Phi=0$, where $\nabla_{\mathcal{E}}=f_{*} \nabla_{\mathcal{L}}$ and $\nabla_{\mathcal{L}}$ is given by (11) (remark, it would be not so easy to find it directly from (12)):

$$
\mathbf{\Phi}=\frac{1}{2}\left(\begin{array}{cc}
e^{-\int \omega}+e^{-\int \omega^{*}} & e^{-\int \omega}-e^{-\int \omega^{*}} \\
\frac{1}{\xi}\left(e^{-\int \omega}-e^{-\int \omega^{*}}\right) & \frac{1}{\xi}\left(e^{-\int \omega}+e^{-\int \omega^{*}}\right)
\end{array}\right),
$$

where $\omega^{*}:=\iota^{*}(\omega)$ is obtained from $\omega$ by the change $\xi \mapsto-\xi$. We deduce the monodromy:

Proposition 9. The monodromy matrices of the connection $\nabla_{\mathcal{E}}$ given by (12) are the following:

$$
\begin{aligned}
M_{a} & =\frac{1}{2}\left(\begin{array}{ll}
e^{-N_{1}}+e^{-N_{2}} & e^{-N_{1}}-e^{-N_{2}} \\
e^{-N_{1}}-e^{-N_{2}} & e^{-N_{1}}+e^{-N_{2}}
\end{array}\right), \\
M_{b} & =\frac{1}{2}\left(\begin{array}{ll}
e^{-N_{3}}+e^{-N_{4}} & e^{-N_{3}}-e^{-N_{4}} \\
e^{-N_{3}}-e^{-N_{4}} & e^{-N_{3}}+e^{-N_{4}}
\end{array}\right), \quad M_{\gamma_{i}}=\left(\begin{array}{cc}
1 & 0 \\
0 & -1
\end{array}\right) \quad(i=1,2) .
\end{aligned}
$$

Here $\left(N_{1}, N_{2}, N_{3}, N_{4}\right)$ are the periods of $\omega$ as defined in (6). 
Proof. By a direct calculation using the observation that the periods of $\omega^{*}$ are $\left(N_{2}, N_{1}, N_{4}\right.$, $\left.N_{3}\right)$.

Here the connection (12) depends on 6 independent parameters $\tilde{q_{1}}, \tilde{q_{2}}, \lambda_{1}, \lambda_{2}, t, t^{\prime}$, and the monodromy is determined by the 4 periods $N_{i}$. Hence, it is justified to speak about the isomonodromic deformations for this connection. The problem of isomonodromic deformations is easily solved upon an appropriate change of parameters. Firstly, change the representation of $\omega$ : write $\omega=\omega_{0}+\lambda_{1} \omega_{1}+\lambda_{2} \omega_{2}$, where $\omega_{0}=\nu+\lambda_{10} \omega_{1}+\lambda_{02} \omega_{2}$ is chosen with zero $a$-periods, as in the proof of Proposition 5, and assume that $\left(\omega_{1}, \omega_{2}\right)$ is a normalized basis of differentials of first kind on $C$. Secondly, replace the 2 parameters $\tilde{q_{1}}, \tilde{q_{2}}$ by the coordinates $z_{1}[L], z_{2}[L]$ of the class of $L=\mathcal{O}\left(\tilde{q}_{1}+\tilde{q}_{2}-\infty_{+}-\infty_{-}\right)$in $J C$. Thirdly, replace $\left(t, t^{\prime}\right)$ by the period $Z$ of $C$. Then an isomonodromic variety $N_{i}=$ const $(i=1, \ldots, 4)$ is defined, in the above parameters, by the equations

$$
\left(\begin{array}{l}
\lambda_{1} \\
\lambda_{2}
\end{array}\right)=\text { const, } \quad\left(\begin{array}{l}
z_{1}[L] \\
z_{2}[L]
\end{array}\right)+Z\left(\begin{array}{l}
\lambda_{1} \\
\lambda_{2}
\end{array}\right)=\text { const. }
$$

Thus the isomonodromy varieties can be considered as surfaces in the 4-dimensional relative Jacobian $J(\mathcal{C} / \mathcal{H})$ of the universal family of bielliptic curves $\mathcal{C} \rightarrow \mathcal{H}$ over the bielliptic period locus $\mathcal{H}$ introduced in Corollary 1 . The fiber $C_{Z}$ of $\mathcal{C}$ over a point $Z \in \mathcal{H}$ is a genus-2 curve with period $Z$, and $J(\mathcal{C} / \mathcal{H}) \rightarrow \mathcal{H}$ is the family of the Jacobians of all the curves $C_{Z}$ as $Z$ runs over $\mathcal{H}$. The isomonodromy surfaces $S_{\lambda_{1}, \lambda_{2}, \mu_{1}, \mu_{2}}$ in $J(\mathcal{C} / \mathcal{H})$ depend on 4 parameters $\lambda_{i}, \mu_{i}$. Every isomonodromy surface is a cross-section of the projection $J(\mathcal{C} / \mathcal{H}) \rightarrow \mathcal{H}$ defined by

$$
S_{\lambda_{1}, \lambda_{2}, \mu_{1}, \mu_{2}}=\left\{(Z,[\mathcal{L}]) \mid Z \in \mathcal{H},[\mathcal{L}] \in J C_{Z},\left(\begin{array}{c}
z_{1}[L] \\
z_{2}[L]
\end{array}\right)=-Z\left(\begin{array}{c}
\lambda_{1} \\
\lambda_{2}
\end{array}\right)+\left(\begin{array}{c}
\mu_{1} \\
\mu_{2}
\end{array}\right)\right\} .
$$

\section{Elementary transforms of rank-2 vector bundles}

In this section, we will recall basic facts on elementary transforms of vector bundles in the particular case of rank 2 , the only one needed for application to the underlying vector bundles of the direct image connection in the next section. The impact of the elementary transforms is twofold. First, they provide a tool of identification of vector bundles. If we are given a vector bundle $\mathcal{E}$ and if we manage to find a sequence of elementary transforms which connect $\mathcal{E}$ to some "easy" vector bundle $\mathcal{E}_{0}$ (like $\mathcal{O} \oplus \mathcal{O}(-p)$ for a point $p$ ), we provide an explicit construction of $\mathcal{E}$ and at the same time we determine, or identify $\mathcal{E}$ via this construction. Second, the elementary transforms permit to change the vector bundle endowed with a connection without changing the monodromy of the connection. The importance of such applications is illustrated in the article [7], in which the authors prove that any irreducible representation of the fundamental group of a Riemann surface with punctures can be realized by a logarithmic connection on a semistable vector bundle of degree 0 (see Theorem 2). On one hand, this is a far-reaching generalization of Bolibruch's result [1] which affirms the solvability of the Riemann-Hilbert problem over the Riemann sphere with punctures, and on the other hand, this theorem gives rise to a map from the moduli space of connections to the moduli space of vector bundles, for only the class of semistable vector bundles has a consistent moduli theory. We will illustrate this feature of elementary transforms allowing us to roll between stable, semistable and unstable bundles in the next section.

Let $E$ be a curve. As before, we identify locally free sheaves on $E$ with associated vector bundles. Let $\mathcal{E}$ be a rank-2 vector bundle on $E, p$ a point of $E, \mathcal{E}_{\mid p}=\mathcal{E} \otimes \mathbb{C}_{p}$ the fiber of $\mathcal{E}$ at $p$. Here $\mathbb{C}_{p}$ is the sky-scraper sheaf whose only nonzero stalk is the stalk at $p$, equal to the 1-dimensional vector space $\mathbb{C}$. We emphasize that $\mathcal{E}_{\mid p}$ is a $\mathbb{C}$-vector space of dimension 2 , not 
to be confused with the stalk $\mathcal{E}_{p}$ of $\mathcal{E}$ at $p$, the latter being a free $\mathcal{O}_{p}$-module of rank 2 . Let $e_{1}, e_{2}$ be a basis of $\mathcal{E}_{\mid p}$. We extend $e_{1}, e_{2}$ to sections of $\mathcal{E}$ in a neighborhood of $p$, keeping for them the same notation. We define the elementary transforms $\mathcal{E}^{+}$and $\mathcal{E}^{-}$of $\mathcal{E}$ as subsheaves of $\mathcal{E} \otimes \mathbb{C}(E) \simeq \mathbb{C}(E)^{2}$, in giving their stalks at all the points of $E$ :

$$
\begin{aligned}
& \mathcal{E}^{-}=e l m_{p, e_{2}}^{-}(\mathcal{E}), \quad \mathcal{E}_{p}^{-}=\mathcal{O}_{p} \tau_{P} e_{1}+\mathcal{O}_{p} e_{2}, \\
& \mathcal{E}^{+}=e l m_{p, e_{1}}^{+}(\mathcal{E}), \quad \mathcal{E}_{p}^{+}=\mathcal{O}_{p} \frac{1}{\tau_{P}} e_{1}+\mathcal{O}_{p} e_{2}, \\
& \mathcal{E}_{z}^{ \pm}=\mathcal{E}_{z}, \quad \forall z \in E \backslash\{p\},
\end{aligned}
$$

where $\tau_{P}$ denotes a local parameter at $p$. The thus obtained sheaves are locally free of rank 2 . They fit into the exact triples:

$$
0 \rightarrow \mathcal{E}^{-} \rightarrow \mathcal{E} \stackrel{\gamma}{\longrightarrow} \mathbb{C}(p) \rightarrow 0, \quad 0 \rightarrow \mathcal{E} \rightarrow \mathcal{E}^{+} \rightarrow \mathbb{C}(p) \rightarrow 0 .
$$

Remark, that the surjection $\gamma$ restricted to $\mathcal{E}_{\mid p}$ is a projection parallel to the $e_{2}$ axis; this is the reason for which we included in the notation of $\mathrm{elm}^{-}$its dependence on $e_{2}$. Thus, if we vary $e_{1}$, in keeping $e_{2}$ (or in keeping the proportionality class of $e_{2}$ ), the isomorphism class of elm $_{e_{2}}^{-}$ will not change, but it can change if we vary the proportionality class $\left[e_{2}\right]$ in the projective line $\mathbb{P}\left(\mathcal{E}_{\mid p}\right)$.

For degrees, we have $\operatorname{deg} \mathcal{E}^{ \pm}=\operatorname{deg} \mathcal{E} \pm 1$. We can give a more precise version of this equality in terms of the determinant line bundles: $\operatorname{det} \mathcal{E}^{ \pm}=\operatorname{det} \mathcal{E}( \pm p)$. Here and further on, given a line bundle $\mathcal{L}$ and a divisor $D=\sum n_{i} p_{i}$ on $E$, we denote by $\mathcal{L}(D)$ (" $\mathcal{L}$ twisted by $D$ ") the following line bundle, defined as a sheaf by its stalks at all the points of $E$ : $\mathcal{L}(D)_{z}=\mathcal{L}_{z}$ if $z$ is not among the $p_{i}$, and $\mathcal{L}(D)_{p_{i}}=\tau_{p_{i}}^{-n_{i}} \mathcal{L}_{z}$. For example, the regular sections of $\mathcal{L}(p)$ can be viewed as meromorphic sections of $\mathcal{L}$ with at most simple pole at $p$, whilst the regular sections of $\mathcal{L}(-p)$ are regular sections of $\mathcal{L}$ vanishing at $p$. For the degree of a twist, we have $\operatorname{deg} \mathcal{L}(D)=\operatorname{deg} \mathcal{L}+\operatorname{deg} D=\operatorname{deg} \mathcal{L}+\sum n_{i}$, so that $\operatorname{deg} \mathcal{L}( \pm p)=\operatorname{deg} \mathcal{L} \pm 1$.

A similar notion of twists applies to higher-rank bundles $\mathcal{E}$ : the twist $\mathcal{E}(D)$ can be defined either as $\mathcal{E} \otimes \mathcal{O}(D)$, or via the stalks in replacing $\mathcal{L}$ by $\mathcal{E}$ in the above definition. For degrees, we have $\operatorname{deg} \mathcal{E}(D)=\operatorname{deg} \mathcal{E}+\operatorname{rk} \mathcal{E} \cdot \operatorname{deg} D$. Coming back to $\mathrm{rk} \mathcal{E}=2$ and twisting $\mathcal{E}$ by $\pm p$, we obtain some more exact triples:

$$
0 \rightarrow \mathcal{E}^{+} \rightarrow \mathcal{E}(p) \rightarrow \mathbb{C}(p) \rightarrow 0, \quad 0 \rightarrow \mathcal{E}(-p) \rightarrow \mathcal{E}^{-} \rightarrow \mathbb{C}(p) \rightarrow 0 .
$$

They are easily defined via stalks, as $\mathcal{E}(p)=\mathcal{E} \otimes \mathcal{O}_{E}(p)$ is spanned by $\frac{1}{\tau_{P}} e_{1}, \frac{1}{\tau_{P}} e_{2}$ at $p$, and $\mathcal{E}(-p)$ by $\tau_{P} e_{1}, \tau_{P} e_{2}$.

A basis-free description of elms can be given as follows: Let $W \subset \mathcal{E}_{\mid p}$ be a 1-dimensional vector subspace. Then $\operatorname{elm}_{(p, W)}^{-}(\mathcal{E})$ is defined as the kernel of the composition of natural maps $\mathcal{E} \rightarrow \mathcal{E}_{\mid p} \rightarrow \mathcal{E}_{\mid p} / W$ (here $\mathcal{E}_{\mid p}, \mathcal{E}_{\mid p} / W$ are considered as sky-scraper sheaves, i.e. vector spaces placed at $p$ ). The positive elm is defined via the duality:

$$
e l m_{p, W}^{+}(\mathcal{E}):=\left(e l m_{p, W^{\perp}}^{-}\left(\mathcal{E}^{\vee}\right)\right)^{\vee} .
$$

To set a correspondence with the previous notation, we write:

$$
\text { elm }{ }_{p, e_{1}}^{+}(\mathcal{E})=\operatorname{elm} m_{p, \mathbb{C} e_{1}}^{+}(\mathcal{E}), \quad \operatorname{elm}_{p, e_{2}}^{-}(\mathcal{E})=\operatorname{elm}_{p, \mathbb{C} e_{2}}^{-}(\mathcal{E})
$$

One can also define $\mathrm{elm}^{+}$as an appropriate $\mathrm{elm}^{-}$, applied not to $\mathcal{E}$, but to $\mathcal{E}(p)$ :

$$
e l m_{p, e_{1}}^{+}=e l m_{p, e_{1}}^{-}(\mathcal{E}(p)) .
$$



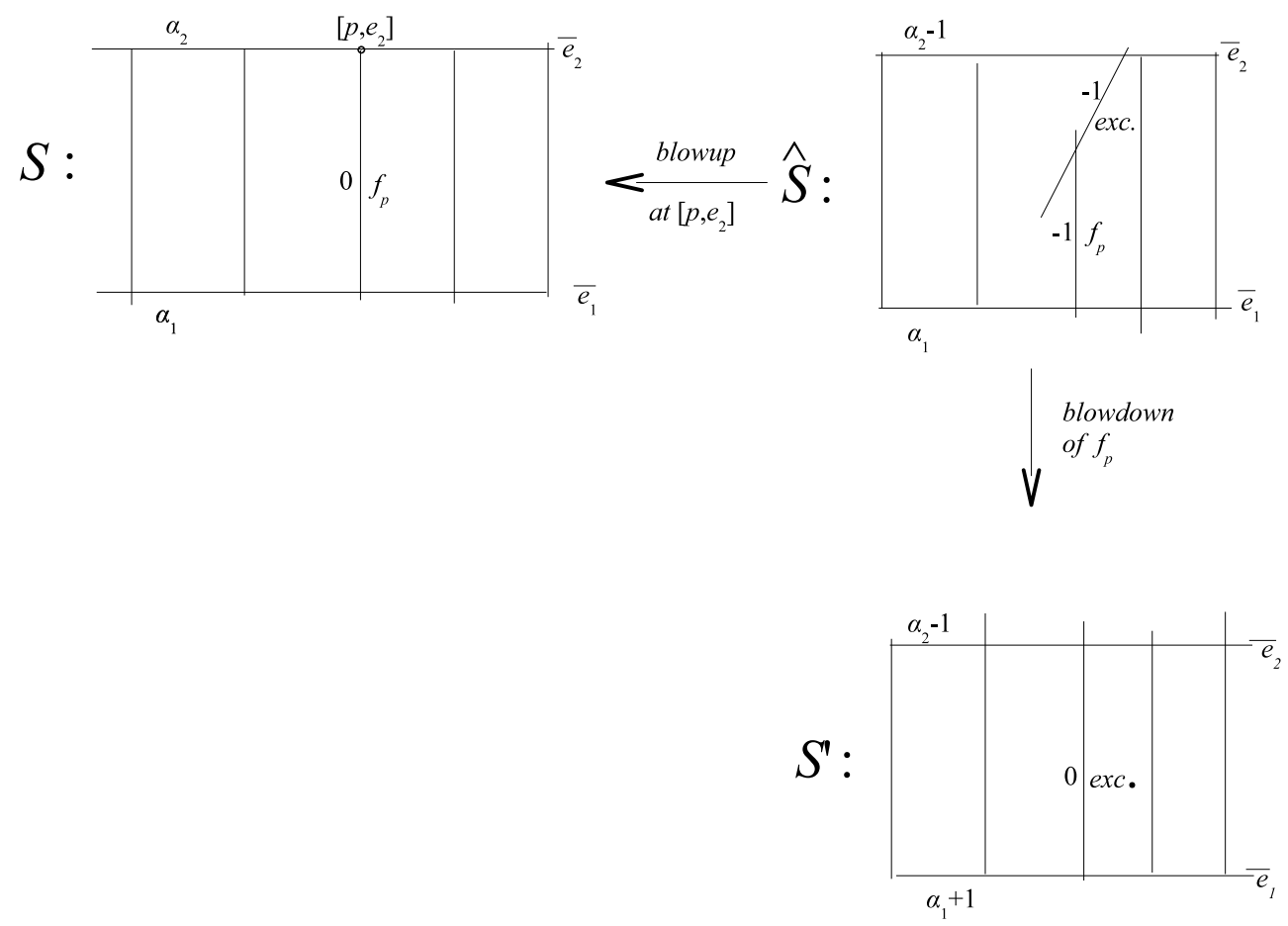

Figure 3. Decomposition of $\mathrm{elm}^{-}$in a blowup followed by a blowdown.

We will now interpret the elementary transforms in terms of ruled surfaces. For a vector bundle $\mathcal{E}$ over $E$, we denote by $\mathbb{P}(\mathcal{E})$ the projectivization of $\mathcal{E}$, whose fiber over $z \in E$ is the projective line $\mathbb{P}\left(\left.\mathcal{E}\right|_{z}\right)$ parameterizing vector lines in $\mathcal{E}_{\mid z}$. It has a natural projection $\mathbb{P}(\mathcal{E}) \rightarrow E$ with fibers isomorphic to $\mathbb{P}^{1}$ and is therefore called a ruled surface. We will see that the elementary transforms of vector bundles correspond to birational maps between associated ruled surfaces which split into the composition of one blowup and one blowdown. The transfer to ruled surfaces allows us to better understand the structure of $\mathcal{E}$, for it replaces all the line subbundles $\mathcal{L} \subset \mathcal{E}$ by cross-sections of the fiber bundle $\mathbb{P}(\mathcal{E}) \rightarrow E$; the latter cross-sections being curves in a surface, we can use the intersection theory on the surface to study them. As an example, we will give a criterion of (semi)stability of $\mathcal{E}$ in terms of the intersection theory on $\mathbb{P}(\mathcal{E})$.

Let us return to the setting of the description of elms via bases. We can assume that $e_{1}$, $e_{2}$ are rational sections of $\mathcal{E}$, regular and linearly independent at $p$. Let $S=\mathbb{P}(\mathcal{E})$ and let $\pi: S \longrightarrow E$ be the natural projection. Then $e_{1}, e_{2}$ define two global cross-sections of $\pi$, which will be denoted $\overline{e_{1}}, \overline{e_{2}}$. If $\mathcal{E}^{-}=\operatorname{elm}_{p, e_{2}}^{-}(\mathcal{E})$, then the natural map $\mathcal{E}^{-} \longrightarrow \mathcal{E}$ gives rise to the birationnal isomorphism of ruled surfaces $S \longrightarrow S^{-}=\mathbb{P}\left(\mathcal{E}^{-}\right)$which splits into the composition of one blowup and one blowdown, as shown on Fig. 3 .

Let $f_{p}$ denote the fiber $\pi^{-1}(p) \simeq \mathbb{P}^{1}$ of $\pi$; we keep the same notation for curves and their proper transforms in birational surfaces. We label some of the curves by their self-intersection; for example, $\left({\overline{e_{1}}}^{2}\right)_{S}=\alpha_{1},\left(f_{p}^{2}\right)_{S}=0,\left(f_{p}^{2}\right)_{\hat{S}}=-1$. For any vector $v \in \mathcal{E}_{\left.\right|_{p}} \backslash\{0\}$, we denote by $[p, v]$ the point of $f_{p}=\mathbb{P}\left(\mathcal{E}_{\left.\right|_{p}}\right)$ which is the vector line spanned by $v$. Remark that the crosssections $\overline{e_{1}}, \overline{e_{2}}$ are disjoint in the neighborhood of $p$ where $e_{1}, e_{2}$ is a basis of $\mathcal{E}$, but $\overline{e_{1}}$ can intersect $\overline{e_{2}}$ at a finite number of points where $e_{1}, e_{2}$ fail to generate $\mathcal{E}$.

The positive elm has a similar description. Basically, as $\mathbb{P}(\mathcal{E}) \simeq \mathbb{P}(\mathcal{E} \otimes L)$ for any invertible sheaf $L$ on $E$, we have $\mathbb{P}(\mathcal{E}) \simeq \mathbb{P}(\mathcal{E}(p))$. Hence, in view of $(18)$, elm ${ }^{+}$and elm ${ }^{-}$have the same representation on the level of ruled surfaces. There exists also an elegant way to define $\mathrm{elm}^{-}$in using $\pi: S \longrightarrow E$ :

$$
\operatorname{elm}_{p, v}^{-}(\mathcal{E})=\pi_{*}\left(I_{S,[p, v]}(1)\right) .
$$


Here, $I_{S,[p, v]}$ is the ideal sheaf of the point $[p, v]$, and $F(1)$ denotes the twist of a sheaf $F$ by $\mathcal{O}_{\mathbb{P}(\mathcal{E}) / E}(1)$. We have the natural exact triple of an ideal sheaf on $S=\mathbb{P}(\mathcal{E})$ :

$$
0 \rightarrow I_{S,[p, v]}(1) \rightarrow \mathcal{O}_{S / E}(1) \rightarrow \mathbb{C}_{[p, v]} \rightarrow 0 .
$$

By a basic property of the tautological sheaf $\mathcal{O}_{S / E}(1)$, we have $\pi_{*} \mathcal{O}_{S / E}(1) \simeq \mathcal{E}$. By applying $\pi_{*}$, we get the exact triple

$$
0 \rightarrow \pi_{*} I_{S,[p, v]}(1) \rightarrow \mathcal{E} \rightarrow \mathbb{C}_{p} \rightarrow 0 .
$$

One can prove that in this way we recover the first exact triple (17).

Now, we will say a few words about the (semi)-stability in terms of ruled surfaces.

Definition 3. A rank-2 vector bundle on a curve $E$ is stable (resp. semistable) if for any line subbundle $\mathcal{L} \subset \mathcal{E}, \operatorname{deg} \mathcal{L}<\frac{1}{2} \operatorname{deg} \mathcal{E}$ (resp., $\operatorname{deg} \mathcal{L} \leq \frac{1}{2} \operatorname{deg} \mathcal{E}$ ), or equivalently, if for any surjection $\mathcal{E} \rightarrow \mathcal{M}$ onto a line bundle $\mathcal{M}$, $\operatorname{deg} \mathcal{M}>\frac{1}{2} \operatorname{deg} \mathcal{E}$ (resp., $\operatorname{deg} \mathcal{M} \geq \frac{1}{2} \operatorname{deg} \mathcal{E}$ ). A vector bundle is called unstable if it is not semistable. It is called strictly semistable if it is semistable, but not stable.

Definition 4. Let $\mathcal{E}$ be a rank-2 vector bundle on a curve $E$. The index of the ruled surface $\pi: S=\mathbb{P}(\mathcal{E}) \longrightarrow E$ is the minimal self-intersection number of a cross-section of $\pi$ :

$$
i(S)=\min \left\{(e)_{S}^{2} \mid e \subset S \text { is a cross-section of } \pi\right\} .
$$

The assertion of the following proposition is well-known, see e.g. [14, p. 55]. For the reader's convenience, we provide a short proof of it.

Proposition 10. $\mathcal{E}$ is stable (resp. semi-stable) iff $i(S)>0$ (resp. $i(S) \geq 0)$.

Proof. The cross-sections of $\mathbb{P}(\mathcal{E}) \longrightarrow E$ are in 1-to-1 correspondence with the exact triples

$$
0 \rightarrow \mathcal{L}_{1} \stackrel{\alpha}{\longrightarrow} \mathcal{E} \stackrel{\beta}{\longrightarrow} \mathcal{L}_{2} \rightarrow 0
$$

where $\mathcal{L}_{1}, \mathcal{L}_{2}$ are line bundles over $E$. The cross-section $e$ associated to such a triple is $\mathbb{P}\left(\mathcal{L}_{1}\right) \subset$ $\mathbb{P}(\mathcal{E})$. It is the zero locus of $\pi^{*} \beta \circ \pi^{*} \alpha \in \operatorname{Hom}\left(\pi^{*} \mathcal{L}_{1}, \pi^{*} \mathcal{L}_{2}\right) \simeq H^{0}\left(S, \pi^{*}\left(\mathcal{L}_{2} \otimes \mathcal{L}_{1}^{-1}\right)\right)$. Hence, the normal bundle $N_{e / S}$ is isomorphic to $\mathcal{L}_{2} \otimes \mathcal{L}_{1}^{-1}$. The stability (resp. semi-stability) of $\mathcal{E}$ is equivalent to the fact that $\operatorname{deg} \mathcal{L}_{1}<\operatorname{deg} \mathcal{L}_{2}$ (resp. $\operatorname{deg} \mathcal{L}_{1} \leq \operatorname{deg} \mathcal{L}_{2}$ ) for any triple (19). As $\left(e^{2}\right)_{S}=\operatorname{deg} N_{e / S}=\operatorname{deg} \mathcal{L}_{2}-\operatorname{deg} \mathcal{L}_{1}$, this ends the proof.

We will end this section by two lemmas which help to identify vector bundles via the geometry of the associated ruled surfaces.

Lemma 3. Let $\mathcal{E}$ be a rank-2 vector bundles over a curve $X$ such that the associated ruled surface $S=\mathbb{P}(\mathcal{E})$ has two disjoint cross-sections $s_{1}, s_{2}$. Then $\mathcal{E}=\mathcal{L}_{1} \oplus \mathcal{L}_{2}$, where $\mathcal{L}_{i}$ are line subbundles of $\mathcal{E}$ corresponding to $s_{i}: s_{i}=\mathbb{P}\left(\mathcal{L}_{i}\right), i=1,2$. Further, for the self-intersection numbers of $s_{i}$, we have $\left(s_{1}\right)^{2}=-\left(s_{2}\right)^{2}=\operatorname{deg} \mathcal{L}_{2}-\operatorname{deg} \mathcal{L}_{1}$.

Proof. The first assertion is obvious, and the second one follows from the formula for $\left(e^{2}\right)_{S}$ in the proof of Proposition 10, in taking into account that $\mathcal{E}=\mathcal{L}_{1} \oplus \mathcal{L}_{2}$ fits into an exact triple of the form (19).

Lemma 4. Let $\mathcal{E}$ be a rank-2 vector bundle over a curve $X, \mathcal{L}$ a line subbundle of $\mathcal{E}$ and $s=\mathbb{P}(\mathcal{L})$ the associated cross-section of the ruled surface $S=\mathbb{P}(\mathcal{E})$. Let $p \in X,[p, v] \in f_{p}$, where $f_{p}$ denotes the fiber of $S$ over $p$. Let $S^{ \pm}=\mathbb{P}\left(\mathcal{E}^{ \pm}\right)$, where $\mathcal{E}^{ \pm}=$elm ${ }_{p, v}^{ \pm}, \pi^{ \pm}: S \rightarrow S^{ \pm}$ the natural birational map, $s^{ \pm}$the proper transform of $s$ in $S^{ \pm}$under $\pi^{ \pm}$(that is, the closure of $\left.\pi^{ \pm}(s \backslash\{[p, v]\})\right)$, and $\mathcal{L}^{ \pm}$the line subbundle of $\mathcal{E}^{ \pm}$such that $s^{ \pm}=\mathbb{P}\left(\mathcal{L}^{ \pm}\right)$. Then we have: 
(i) If $[p, v] \in s$, then $\left(s^{ \pm}\right)_{S^{ \pm}}^{2}=\left(s^{2}\right)_{S}-1$, $\operatorname{deg} \mathcal{L}^{+}=\operatorname{deg} \mathcal{L}+1$, and $\operatorname{deg} \mathcal{L}^{-}=\operatorname{deg} \mathcal{L}$. Moreover, $\mathcal{L}^{+} \simeq \mathcal{L}(p)$ and $\mathcal{L}^{-} \simeq \mathcal{L}$.

(ii) If $[p, v] \notin s$, then $\left(s^{ \pm}\right)_{S^{ \pm}}^{2}=\left(s^{2}\right)_{S}+1, \operatorname{deg} \mathcal{L}^{+}=\operatorname{deg} \mathcal{L}$, and $\operatorname{deg} \mathcal{L}^{-}=\operatorname{deg} \mathcal{L}-1$. Moreover, $\mathcal{L}^{+} \simeq \mathcal{L}$ and $\mathcal{L}^{-} \simeq \mathcal{L}(-p)$

Proof. The formulas for $\left(s^{ \pm}\right)_{S^{ \pm}}^{2}$ follow from the behavior of the intersection indices as shown on Fig. 3, and those for $\operatorname{deg} \mathcal{L}^{ \pm}$are easily deduced directly from the definition of elementary transforms (16) by choosing for $e_{1}$ or $e_{2}$ a rational trivialization of $\mathcal{L}$.

\section{Underlying vector bundles of direct image connection}

Let us go over again to the setting of Section 4. Consider first the case when $\mathcal{L}$ is the trivial bundle, $\mathcal{L}=\mathcal{O}_{C}$. The following fact is well known:

Lemma 5. Let $f: X \rightarrow Y$ be a finite morphism of smooth varieties of degree 2 and $\Delta$ the class of its branch divisor in $\operatorname{Pic}(Y)$. Then $\Delta$ is divisible by two in $\operatorname{Pic}(Y)$, and there exists $\delta \subset \operatorname{Pic}(Y)$ such that $2 \delta$ is linearly equivalent to $\Delta$ and $f_{*} \mathcal{O}_{X}=\mathcal{O}_{Y} \oplus \mathcal{O}_{Y}(-\delta)$.

Proof. See [11, Section 1].

Applying this lemma to $f: C \rightarrow E$, we find that $f_{*} \mathcal{O}_{C}=\mathcal{O}_{E} \oplus \mathcal{O}_{E}(-\delta)$, where $2 \delta \simeq p_{+}+p_{-}$. This property determines $\delta$ only modulo $E[2]$, but as we saw in Section $4, \mathcal{O}_{E}(-\delta)$ is trivialized by a section $\xi$ over $E \backslash\{\infty\}$, thus $\delta=\infty$ and $f_{*} \mathcal{O}_{C}=\mathcal{O}_{E} \oplus \mathcal{O}_{E}(-\infty)$. We deduce:

Proposition 11. If $\mathcal{L}=\mathcal{O}_{C}$, then the direct image connection $\nabla_{\mathcal{E}}=f_{*}\left(\nabla_{\mathcal{L}}\right)$, determined by formula (12), is a logarithmic connection on the vector bundle $\mathcal{E}_{0}=\mathcal{O}_{E} \oplus \mathcal{O}_{E}(-\infty)$ with two poles at $p_{+}, p_{-}$.

Let now $\mathcal{L}$ be an arbitrary line bundle over $C$ of degree $0 . \quad$ By continuity, $\operatorname{deg} f_{*} \mathcal{L}=$ $\operatorname{deg} f_{*} \mathcal{O}_{C}=-1$. To determine $f_{*} \mathcal{L}$, we use the following lemma:

Lemma 6. Let $X$ be a nonsingular curve, $p$ a point in $X$, and $z$ a local parameter at $p$. Let $\mathcal{E}$ be a rank-2 vector bundle on $X$ with a meromorphic connection $\nabla$, regular at $p$. Let $s_{1}, s_{2}$ be a pair of meromorphic sections of $\mathcal{E}$, linearly independent over $\mathbb{C}(X)$ and $\mathcal{F}=\left\langle s_{1}, s_{2}\right\rangle$ the subsheaf of $\mathcal{E} \otimes \mathbb{C}(X)$ generated by $s_{1}, s_{2}$ as a $\mathcal{O}_{X}$-module. Let $\mathcal{A}$ be the matrix of $\nabla$ with respect to the $\mathbb{C}(X)$-basis $s_{1}, s_{2}$ and $A=\operatorname{res}_{z=0} \mathcal{A}$. Assume that $\mathcal{E}_{\mid p}$ has a basis $v_{1}, v_{2}$ consisting of eigenvectors of $A$. Then $v_{1}, v_{2}$ extend to a basis of the stalk $\mathcal{E}_{p}$, the corresponding eigenvalues $n_{1}, n_{2}$ of $A$ are integers and we have the following relations between the stalks of subsheaves of $\mathcal{E} \otimes \mathbb{C}(X)$ at $p:$

$$
\begin{aligned}
& \mathcal{E}_{p}=\left\langle v_{1}, v_{2}\right\rangle, \quad \mathcal{F}_{p}=\left\langle z^{n_{1}} v_{1}, z^{n_{2}} v_{2}\right\rangle, \\
& \text { if } n_{1}=1, \quad n_{2}=0, \quad \mathcal{E}_{p}=\operatorname{elm}_{p, v_{1}}^{+}\left(F_{p}\right), \\
& \text { if } n_{1}=-1, \quad n_{2}=0, \quad \mathcal{E}_{p}=\operatorname{elm}_{p, v_{2}}^{-}\left(F_{p}\right), \\
& \text { if } n_{1}=n_{2}, \quad \mathcal{E}_{p}=\left(F\left(n_{1}\right)\right)_{p} .
\end{aligned}
$$

Proof. Straightforward.

Let us apply this lemma to the connection $\nabla_{\mathcal{E}}$, given by formula $(12)$ in the basis $(1, \xi)$. We have: $\mathcal{E}_{p} \neq \mathcal{F}_{p} \Longleftrightarrow p \in\left\{q_{1}, q_{2}, \infty\right\}$,

$$
A_{i}=\operatorname{Res}_{q_{i}} \mathcal{A}=\left(\begin{array}{cc}
\frac{1}{2} & \frac{\xi_{1}}{2} \\
\frac{1}{2 \xi_{1}} & \frac{1}{2}
\end{array}\right) \quad(i=1,2), \quad A_{\infty}=\operatorname{Res}_{\infty} \mathcal{A}=\left(\begin{array}{cc}
-1 & -\frac{\xi_{1}+\xi_{2}}{2} \\
0 & -2
\end{array}\right) .
$$


We list the eigenvectors $v_{j}^{(i)}, v_{j}^{(\infty)}$ together with the respective eigenvalues for the matrices $A_{i}$, $A_{\infty}$ :

$$
\begin{array}{ll}
v_{1}^{(i)}=\left(\begin{array}{c}
-\xi_{i} \\
1
\end{array}\right), \quad \eta_{1}^{i}=0, \quad v_{2}^{(i)}=\left(\begin{array}{c}
\xi_{i} \\
-1
\end{array}\right), \quad \eta_{2}^{i}=-1, \\
v_{1}^{(\infty)}=\left(\begin{array}{c}
1 \\
0
\end{array}\right), \quad \eta_{1}^{\infty}=1, \quad v_{2}^{(\infty)}=\left(\begin{array}{c}
-\frac{\xi_{1}+\xi_{2}}{2} \\
1
\end{array}\right), \quad \eta_{2}^{\infty}=-2 .
\end{array}
$$

Applying Lemma 6 (twice at $\infty$ ), we obtain the following corollary:

Corollary 2. Let $\mathcal{L}=\mathcal{O}_{C}\left(\tilde{q}_{1}+\tilde{q}_{2}-\infty_{+}-\infty_{-}\right), \tilde{q}_{i}=\left(\xi_{i}, y_{i}\right), q_{i}=f\left(\tilde{q}_{i}\right), i=1,2$, as in Proposition 7 , and let $v_{j}^{(i)}$ be the eigenvectors of $A_{i}$ as above. Then

$$
\mathcal{E}=\operatorname{elm}_{q_{1}, v_{2}^{(1)}}^{+} \operatorname{elm}_{q_{2}, v_{2}^{(2)}}^{+}\left(\mathcal{E}_{0}(-\infty)\right)
$$

Remark 1. Note that though the sheaf-theoretic direct image $f_{*} \mathcal{L}$ does not depend on the choice of a connection $\nabla_{\mathcal{L}}$ on $\mathcal{L}$, our method of computation of $f_{*} \mathcal{L}$, given by Corollary 2, uses the direct image connection $\nabla_{\mathcal{E}}=f_{*} \nabla_{\mathcal{L}}$ for some $\nabla_{\mathcal{L}}$.

Proposition 12. For generic $\mathcal{L} \in \operatorname{Pic}(C)$, the rank-2 vector bundle $\mathcal{E}$ is stable.

Proof. Starting from the ruled surface $S_{0}=\mathbb{P}\left(\mathcal{E}_{0}\right)=\mathbb{P}\left(\mathcal{O}_{E} \oplus \mathcal{O}_{E}(-\infty)\right)$, we apply two elementary transforms $S_{0} \rightarrow S_{1} \rightarrow S_{2}=\mathbb{P}(\mathcal{E})$, and we have to prove that any cross-section of $S_{2}$ has strictly positive self-intersection, provided that $\tilde{q}_{i}=\left(\xi_{i}, y_{i}\right)$ are sufficiently generic. For a rational section $s$ of $\mathcal{E}_{0}$, let us denote by $\bar{s}$ the associated cross-section of $S_{0} . S_{0}$ is characterized by the existence of two distinguished sections $\bar{s}_{1}, \bar{s}_{2}$ associated to $s_{1}=1, s_{2}=\xi$ with self-intersections $\bar{s}_{1}^{2}=-1, \bar{s}_{2}^{2}=1$, and we have the relations $\bar{s}_{1} \bar{s}_{2}=0, \bar{s}_{2} \sim \bar{s}_{1}+f_{\infty}$, where $f_{p}=\pi^{-1}(p)$ is the fiber of the structure projection $\pi: S_{0} \longrightarrow E$. When there is no risk of confusion, we will keep the same notation for curves and their proper transforms in birational surfaces. Any cross-section $\bar{s}$ is linearly equivalent to $\bar{s}_{1}+f_{p_{1}}+\cdots+f_{p_{r}}$ for some points $p_{1}, \ldots, p_{r}$ in $E$, and $\bar{s}^{2}=2 r+1$. In particular, $i\left(S_{0}\right)=-1$, attained on $\bar{s}_{1}$. Remark that $\bar{s}_{0}$ is rigid, whilst $\bar{s}_{1}$ moves in a pencil $\left|\bar{s}_{1}+f_{\infty}\right|$. Let us apply $\operatorname{elm}_{q_{1}, v_{2}^{(1)}}$. First, we blow up $P_{1}=\left[q_{1}, v_{2}^{(1)}\right]$. Let $\bar{e}_{1}$ be the corresponding $(-1)$-curve and $\hat{S_{0}}$ the blown up surface. For the self-intersection numbers of the cross-sections, we have the following relations: $\left(\bar{s}^{2}\right)_{\hat{S}_{0}}=\left(\bar{s}^{2}\right)_{S_{0}}$ if $P_{1} \notin \bar{s}$ and $\left(\bar{s}^{2}\right)_{\hat{S}_{0}}=\left(\bar{s}^{2}\right)_{S_{0}}-1$ if $P_{1} \in \bar{s}$. Hence, $\hat{S}_{0}$ has only one cross-section for each one of the self-intersection numbers $-1,0$, and $\left(\bar{s}^{2}\right)_{S_{0}} \geq 1$ for all the other cross-sections. The cross-section with self-intersection -1 is $\bar{s}_{1}$ and the one with self-intersection 0 is the proper transform of the unique member $\bar{s}_{P_{1}}$ of the pencil $\left|\bar{s}_{1}+f_{\infty}\right|$ on $S_{0}$ going through $P_{1}$, see Fig. 4 . The next step is the blowdown of $f_{q_{1}} \subset \hat{S}_{0}$. The self-intersection number of all the cross-sections of $\hat{S}_{0} \rightarrow E$ that meet $f_{q_{1}}$ goes up by 1 . We conclude that $S_{1}=\mathbb{P}\left(e l m_{q_{1}, v_{2}^{1}}^{+}\left(\mathcal{E}_{0}\right)\right)$ has two cross-sections $\bar{s}_{P_{1}}, \bar{s}_{1}$ with square 0 , and $\left(\bar{s}^{2}\right)_{S_{1}} \geq 2$ for any other cross-section of $S_{1}$. In the language of vector bundles, this means that $\mathcal{E}_{1}$ is the direct sum of two line bundles of degree 0 . More precisely, $\mathcal{E}_{1}=\mathcal{O}_{E} \oplus \mathcal{O}_{E}\left(q_{1}-\infty\right)$ by Lemma 3 , the first summand corresponding to $\bar{s}_{1}$ and the second one to $\bar{s}_{P_{1}}$.

The second elementary transform is performed at $P_{2} \in S_{1}$. As $P_{2} \notin \bar{s}_{P_{1}} \cup \bar{s}_{1}$, the minimal selfintersection number of a cross-section in $S_{1}$ passing through $P_{2}$ is 2 . The elementary transform decreases by 1 the self-intersection of such cross-sections and increases by 1 the self-intersection of all other cross-sections (Lemma 4). Hence, $i\left(S_{2}\right)=1$, the value attained on many cross-sections, for example, $\bar{s}_{P_{2}}, \bar{s}_{P_{1}}, \bar{s}_{1}$. This ends the proof.

Theorem 1 (Atiyah, [2]). For any line bundle $\mathcal{N}$ of odd degree over an elliptic curve $E$, there exists one and only one stable rank-2 vector bundle on $E$ with determinant $\mathcal{N}$. 


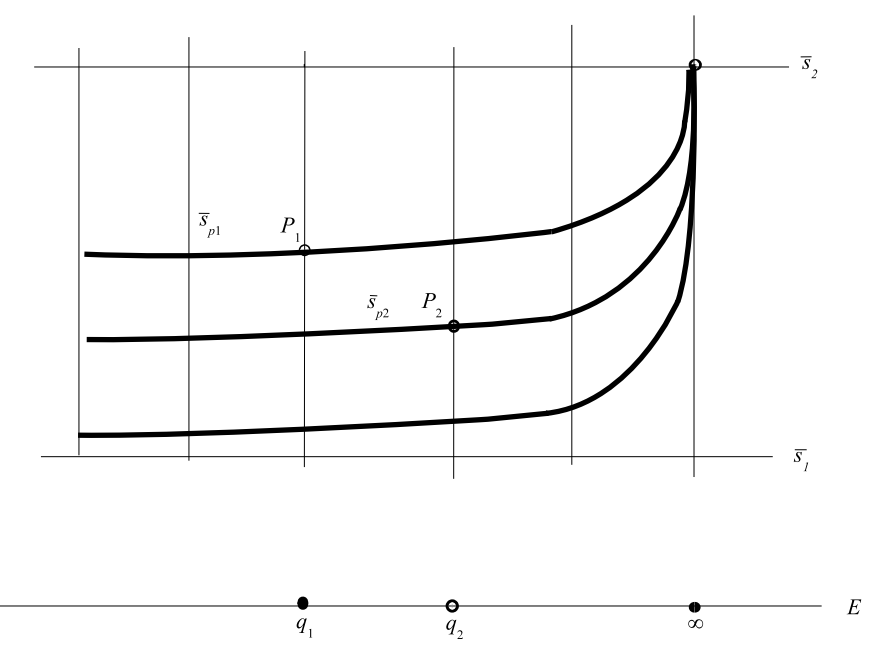

Figure 4. The ruled surface $S_{0}$. The pencil $\left|\bar{s}_{1}+f_{\infty}\right|$ has a unique member passing through $P_{i}$ for each $i=1,2$.

Using Atiyah's theorem in our case, we have $\operatorname{deg} \mathcal{N}=-1$, so that $\mathcal{N}$ can be represented in the form $\mathcal{N}=\mathcal{O}_{E}(-q)$ for some $q \in E$. $\mathcal{E}$ is obtained as the unique non-trivial extension of vector bundles:

$$
0 \rightarrow \mathcal{O}_{E}(-q) \rightarrow \mathcal{E} \rightarrow \mathcal{O}_{E} \rightarrow 0
$$

Moreover, the correspondence $\mathcal{E} \leftrightarrow q$ identifies the moduli space $\mathcal{M}_{E}^{s}(2,-1)$ of rank-2 stable vector bundles of degree -1 over $E$ with $E$ itself. We deduce:

Corollary 3. Under the above identification $\mathcal{M}_{E}^{s}(2,-1) \simeq E$, the rational map:

$$
\begin{aligned}
& f: J C \rightarrow \mathcal{M}_{E}^{s}(2,-1), \\
& \mathcal{L}=\mathcal{O}_{C}\left(\tilde{q_{1}}+\tilde{q_{2}}-\infty_{+}-\infty_{-}\right) \mapsto f_{*}(\mathcal{L})
\end{aligned}
$$

can be given by

$$
\left[\tilde{q_{1}}+\tilde{q_{2}}-\infty_{+}-\infty_{-}\right] \mapsto\left[q_{1}+q_{2}-2 \infty\right]
$$

Now we go over to the nongeneric line bundles $\mathcal{L}$. The direct image $f_{*} \mathcal{L}$ can be unstable for special $\mathcal{L}$. This may happen when either the argument of Proposition 12 does not work anymore, or when formulas (12)-(15) are not valid. We list the cases which need a separate analysis in the next proposition.

Proposition 13. Let $\mathcal{L}=\mathcal{O}_{C}\left(\tilde{q_{1}}+\tilde{q_{2}}-\infty_{+}-\infty_{-}\right), \mathcal{E}=f_{*}(\mathcal{L}), \mathcal{E}_{0}=f_{*} \mathcal{O}_{C}$, as above. Whenever $\tilde{q}_{i}$ is finite, it will be represented by its coordinates: $\tilde{q}_{i}=\left(\xi_{i}, y_{i}\right)$. The following assertions hold:

(a) If $\tilde{q_{1}}+\tilde{q_{2}}$ is a divisor in the hyperelliptic linear series $g_{2}^{1}(C)$ (that is $\xi_{1}=\xi_{2}, y_{1}=-y_{2}$, or $\left.\left\{\tilde{q}_{1}, \tilde{q}_{2}\right\}=\left\{\infty_{+}, \infty_{-}\right\}\right)$, then $\mathcal{E} \simeq \mathcal{E}_{0}$, and hence $\mathcal{E}$ is unstable.

(b) If $\tilde{q_{1}}=\tilde{q_{2}} \neq \infty_{ \pm}$, then $\mathcal{E} \simeq \mathcal{O}_{E}(-\infty) \oplus \mathcal{O}_{E}\left(2 q_{1}-2 \infty\right)$ is unstable.

(c) If $\tilde{q}_{i}=\infty_{ \pm}$for at least one value $i \in\{1,2\}$, then $\mathcal{E} \simeq \mathcal{O}_{E}\left(-2 \infty+q_{3-i}\right) \oplus \mathcal{O}_{E}$ is unstable.

(d) If $\tilde{q}_{i}=\tilde{p}_{ \pm}$for exactly one value $i \in\{1,2\}$, then $\mathcal{E}$ is a stable bundle of degree -1 with $\operatorname{det} \mathcal{E} \simeq \mathcal{O}_{E}\left(q_{3-i}+p_{ \pm}-3 \infty\right)$. 
Proof. (a) In this case, $s_{p_{1}}=s_{p_{2}}, \tilde{q_{1}}+\tilde{q_{2}} \sim \infty_{+}+\infty_{-}$, then $\mathcal{L} \simeq \mathcal{O}_{C}$, and $\mathcal{E} \simeq \mathcal{E}_{0}$.

(b) Let, for example, $i=1$. Then $\mathcal{L}=\mathcal{O}_{C}\left(\tilde{q_{1}}+\tilde{q_{2}}-\infty_{+}-\infty_{-}\right)$degenerates to $\mathcal{L}=$ $\mathcal{O}_{C}\left(2 \tilde{q_{1}}-\infty_{+}-\infty_{-}\right)$. In this case, the matrix of a regular connection on $\mathcal{L}$ in the rational basis 1 of $\mathcal{L}=\mathcal{O}_{C}\left(2 \tilde{q_{1}}-\infty_{+}-\infty_{-}\right) \hookrightarrow \mathcal{O}_{C}\left(2 \tilde{q_{1}}\right) \hookleftarrow \mathcal{O}_{C} \ni 1$ is a rational 1-form with residues 2 at $\tilde{q}_{1}$ and -1 at points $\infty_{ \pm}$. Such a 1 -form can be written by the same formula $\omega=\frac{1}{2}\left(\frac{y+y_{1}}{\xi-\xi_{1}}+\right.$ $\left.\frac{y+y_{2}}{\xi-\xi_{2}}\right) \frac{\mathrm{d} \xi}{y}+\lambda_{1} \frac{\mathrm{d} \xi}{y}+\lambda_{2} \frac{\xi \mathrm{d} \xi}{y}$, as in the general case, but now we substitute $\xi_{2}=\xi_{1}, y_{2}=y_{1}$ in it:

$$
\omega=\frac{y+y_{1}}{\xi-\xi_{1}} \frac{\mathrm{d} \xi}{y}+\lambda_{1} \frac{\mathrm{d} \xi}{y}+\lambda_{2} \frac{\xi \mathrm{d} \xi}{y} .
$$

Assume that $\xi_{1}=\xi_{2} \neq 0$; the case when $\tilde{q}_{1}=\tilde{q}_{2}=\tilde{p}_{ \pm}$should be treated separately as in (d). Then we get the matrix $A$ of $\nabla_{\mathcal{E}}$ in substituting $\xi_{2}=\xi_{1}, y_{2}=y_{1}, x_{2}=x_{1}$ into formulas (12)-(15). We obtain the following residues:

$$
\begin{array}{ll}
\operatorname{Res}_{p^{ \pm}} A & =\left(\begin{array}{cc}
0 & 0 \\
\frac{1}{2} \frac{\left(y_{1} \pm y_{0}\right) \xi_{1}}{\left(x_{1}-t^{\prime}\right)} \pm \frac{\lambda_{1}}{2 y_{0}} & \frac{1}{2}
\end{array}\right), \quad \operatorname{Res}_{q_{1}} A=\left(\begin{array}{cc}
1 & \xi_{1} \\
\xi_{1} & 1
\end{array}\right), \\
\operatorname{Res}_{\infty} A & =\operatorname{Res}_{u=0} A=\left(\begin{array}{cc}
-1 & -\xi_{1} \\
0 & -2
\end{array}\right) .
\end{array}
$$

As in the proof of Proposition 12, we can describe $\mathcal{E}$ as the result of two successive positive elm's applied to $\mathcal{E}_{0}(-\infty)$. In contrast to the general case, considered in Lemma 6 , the second elm has for its center the point $\tilde{P}_{1}=\bar{s}_{P_{1}} \cap \tilde{f_{q_{1}}} \subset S_{1}$, where $\tilde{f_{q_{1}}}$ is the fiber of $S_{1} \rightarrow E$ over $q_{1}$. As $\left(\bar{s}_{P_{1}}\right)_{S_{1}}^{2}=0$, the resulting surface $S_{2}$ has a cross-section with self-intersection -1 , thus $i\left(S_{2}\right)=-1$, and consequently $\mathcal{E}$ is unstable. Applying Lemmas 3 and 4 , we can identify it with $\mathcal{O}_{E}(-\infty) \oplus \mathcal{O}_{E}\left(2 q_{1}-2 \infty\right)$.

(c) Let, for example, $\tilde{q_{2}}=\infty_{-}$. Then $\mathcal{L}$ degenerates to $\mathcal{O}_{C}\left(\tilde{q_{1}}-\infty_{+}\right)$, and we can again write the connection in the same way as in the previous case. $\mathcal{E}$ is obtained from $\mathcal{E}_{0}(-\infty)$ by 2 positive elms. From Lemmas 3 and 4 , we deduce that $\mathcal{E} \simeq \mathcal{O}_{E}\left(-2 \infty+q_{1}\right) \oplus \mathcal{O}_{E}$.

(d) One of the points $\tilde{p}_{ \pm}$collides with $\tilde{q}_{i}$. This corresponds to $\xi_{i}=0$. So, we assume that $\xi_{2}=0, \xi_{1} \neq 0\left(\tilde{q_{2}}=\tilde{p}_{+}\right)$. Hence, the 1 -form of the connection can be written as follows:

$$
\omega=\frac{1}{2}\left(\frac{y+y_{0}}{\xi}+\frac{y+y_{1}}{\xi-\xi_{1}}\right) \frac{\mathrm{d} \xi}{y}+\lambda_{1} \frac{\mathrm{d} \xi}{y}+\lambda_{2} \frac{\xi \mathrm{d} \xi}{y} .
$$

The matrix $A$ is given by

$$
A=\left(\begin{array}{cc}
-\frac{1}{2}\left(\frac{1}{2} \frac{y+y_{1}}{x_{1}-x}+\frac{y+y_{0}}{t^{\prime}-x}+\lambda_{2}\right) \frac{\mathrm{d} x}{y} & -\frac{1}{2}\left(\frac{1}{2} \frac{\left(y+y_{1}\right) \xi_{1}}{x_{1}-x}+\lambda_{1}\right) \frac{\mathrm{d} x}{y} \\
-\frac{1}{2}\left(\frac{1}{2} \frac{\left(y+y_{1}\right) \xi_{1}}{\left(x_{1}-x\right)\left(t^{\prime}-x\right)}+\frac{\lambda_{1}}{t^{\prime}-x}\right) \frac{\mathrm{d} x}{y} & -\frac{1}{2}\left(\frac{1}{2}\left(\frac{y+y_{1}}{x_{1}-x}+\frac{y+y_{0}}{t^{\prime}-x}\right)+\lambda_{2}+\frac{y}{t^{\prime}-x}\right) \frac{\mathrm{d} x}{y}
\end{array}\right) .
$$

Its residues at finite points are:

$$
\begin{aligned}
\operatorname{Res}_{p_{+}} A & =\left(\begin{array}{cc}
\frac{1}{2} & 0 \\
\frac{1}{4} \frac{\left(y_{0}+y_{1}\right) \xi_{1}}{\left(x_{1}-t^{\prime}\right)}+\frac{\lambda_{1}}{2 y_{0}} & 1
\end{array}\right), \quad \operatorname{Res}_{p_{-}} A=\left(\begin{array}{cc}
0 & 0 \\
\frac{1}{4} \frac{\left(y_{1}-y_{0}\right) \xi_{1}}{\left(x_{1}-t^{\prime}\right)}-\frac{\lambda_{1}}{2 y_{0}} & \frac{1}{2}
\end{array}\right), \\
\operatorname{Res}_{q_{1}} A & =\left(\begin{array}{cc}
\frac{1}{2} & \frac{\xi_{1}}{2} \\
\frac{1}{2 \xi_{1}} & \frac{1}{2}
\end{array}\right) .
\end{aligned}
$$

Here $\mathcal{E}_{0}=\mathcal{O}_{E} \oplus \mathcal{O}_{E}(-\infty)$, the first elm applied to $\mathcal{E}_{0}$ gives $\mathcal{E}_{1}=\mathcal{O}_{E} \oplus \mathcal{O}_{E}\left(p_{+}-\infty\right)$, and the second one transforms $\mathcal{E}_{1}$ into a stable vector bundle $\mathcal{E}_{2}$ which fits into the exact triple

$$
0 \rightarrow \mathcal{O}_{E} \rightarrow \mathcal{E}_{2} \rightarrow \mathcal{O}_{E}\left(q_{1}+p_{+}-\infty\right) \rightarrow 0 .
$$

Thus, the resulting vector bundle $\mathcal{E}=f_{*}(\mathcal{L})=\mathcal{E}_{2}(-\infty)$ behaves exactly as in the general case $\left(\tilde{p_{+}} \neq \tilde{q_{2}}\right)$. 
Next we will discuss Gabber's elementary transforms as defined by Esnault and Viehweg [7]. Gabber's transform of a pair $(\mathcal{E}, \nabla)$, consisting of a vector bundle $\mathcal{E}$ over a curve and a logarithmic connection on $\mathcal{E}$ is another pair $\left(\mathcal{E}^{\prime}, \nabla^{\prime}\right)$, where $\mathcal{E}^{\prime}$ is an elementary transform of $\mathcal{E}$ at some pole $p$ of $\nabla$, and one of the eigenvalues of $\operatorname{Res}_{p} \nabla^{\prime}$ differs by 1 from the respective eigenvalue of $\operatorname{Res}_{p} \nabla$, whilst the other eigenvalues as well as the other residues remain unchanged. We adapt the definition of Esnault-Viehweg to the rank-2 case and to our notation:

Definition 5. Let $\mathcal{E}$ be a rank-2 vector bundle on a curve $X, \nabla$ a logarithmic connection on $\mathcal{E}$, $p \in X$ a pole of $\nabla$, and $\left.v \in \mathcal{E}\right|_{p}$ an eigenvector of the residue $\operatorname{Res}_{p}(\nabla) \in \operatorname{End}\left(\left.\mathcal{E}\right|_{p}\right)$. The Gabber transform $e l m_{p, v}(\mathcal{E}, \nabla)$ is a pair $\left(\mathcal{E}^{\prime}, \nabla^{\prime}\right)$ constructed as follows:

(i) $\mathcal{E}^{\prime}=e \operatorname{elm}_{p, v}^{+}(\mathcal{E})$.

(ii) $\nabla^{\prime}$ is identified with $\nabla$ under the isomorphism $\left.\left.\mathcal{E}\right|_{X-p} \simeq \mathcal{E}^{\prime}\right|_{X-p}$ as a meromorphic connection over $X-p$, and this determines $\nabla^{\prime}$ as a meromorphic connection over $X$.

By a local computation of $\nabla^{\prime}$ at $p$ one proves:

Lemma 7. In the setting of Definition 5, let us complete $v$ to a basis $\left(e_{1}=v, e_{2}\right)$ of $\mathcal{E}$ near $p$, so that $\mathcal{E}_{p}^{\prime}=\mathcal{O}_{p} \cdot \frac{1}{\tau_{p}} v+\mathcal{O}_{p} \cdot e_{2}$ and the matrix $R$ of $\operatorname{Res}_{p}(\nabla)$ has the form $R=\left(\begin{array}{cc}\lambda_{1} & * \\ 0 & \lambda_{2}\end{array}\right)$. Then $\nabla^{\prime}$ is a logarithmic connection on $\mathcal{E}^{\prime}$ and the matrix $R^{\prime}$ of its residue at $p$ computed with respect to the basis $\left(e_{1}^{\prime}, e_{2}^{\prime}\right)=\left(\frac{v}{\tau_{p}}, e_{2}\right)$ of $\mathcal{E}^{\prime}$ has the form $R^{\prime}=\left(\begin{array}{cc}\lambda_{1}-1 & 0 \\ * & \lambda_{2}\end{array}\right)$.

Theorem 2 (Bolibruch-Esnault-Viehweg [1, 7]). Let $\mathcal{E}$ be a rank-r vector bundle on a curve $X, \nabla$ a logarithmic connection on $\mathcal{E}$, and assume that the pair $(\mathcal{E}, \nabla)$ is irreducible in the following sense: $\mathcal{E}$ has no $\nabla$-invariant subbundles $\mathcal{F} \subset \mathcal{E}$. Then there exists a sequence of Gabber's transforms that replaces $(\mathcal{E}, \nabla)$ by another pair $\left(\mathcal{E}^{\prime}, \nabla^{\prime}\right)$, in which $\mathcal{E}^{\prime}$ is a semistable vector bundle of degree 0 and $\nabla^{\prime}$ is a logarithmic connection on $\mathcal{E}^{\prime}$ with the same singular points and the same monodromy as $\nabla$.

We are illustrating this theorem by presenting explicitly one elementary Gabber's transform which transforms our bundle $\mathcal{E}=f_{*} \mathcal{L}$ of degree -1 into a semistable bundle $\mathcal{E}^{\prime}$ of degree 0 :

Proposition 14. Let $\mathcal{E}, \nabla$ be as in Proposition 12. Let $v$ be an eigenvector of $\operatorname{Res}_{p_{+}}(\nabla)$ with eigenvalue $\frac{1}{2}$ (see formula (13)). Then the Gabber transform $\left(\mathcal{E}^{\prime}, \nabla^{\prime}\right)=\operatorname{elm}_{p_{+}, v}^{+}(\mathcal{E}, \nabla)$ satisfies the conclusion of the Bolibruch-Esnault-Viehweg theorem: $\mathcal{E}^{\prime}$ is semistable of degree 0 and $\nabla^{\prime}$ is a logarithmic connection with the same singularities and the same monodromy as $\nabla$. Furthermore, $\mathcal{E}^{\prime} \simeq \mathcal{O}_{E}\left(p_{+}-\infty\right) \oplus \mathcal{O}_{E}\left(q_{1}+q_{2}-2 \infty\right)$.

Proof. By Corollary 2, $\mathcal{E}^{\prime}$ is the result of application of three positive elms to $\mathcal{E}_{0}(-\infty)=$ $\mathcal{O}_{E}(-\infty) \oplus \mathcal{O}_{E}(-2 \infty)$ :

$$
\mathcal{E}^{\prime}=\operatorname{elm}_{p_{+}, v}^{+} \text {elm }{ }_{q_{1}, v_{2}^{(1)}}^{+} \operatorname{elm}_{q_{2}, v_{2}^{(2)}}^{+}\left(\mathcal{E}_{0}(-\infty)\right) .
$$

The surface $S_{0}=\mathbb{P}\left(\mathcal{E}_{0}(-\infty)\right)$ can be decomposed as the open subset $S_{0} \backslash \bar{s}_{1}$ (see Fig. 4 ), which is a line bundle over $E$ with zero section $\bar{s}_{2}$, plus the "infinity section" $\bar{s}_{1}$. The line bundle is easily identified as the normal bundle to $\bar{s}_{2}$ in $S_{0}: S_{0} \backslash \bar{s}_{1} \simeq \mathcal{N}_{\bar{s}_{2} / S_{0}} \simeq \mathcal{O}_{E}(\infty)$. Then the pencil $\left|\bar{s}_{2}\right|=\left|\bar{s}_{1}+f_{\infty}\right|$ is the projective line which naturally decomposes into the affine line $H^{0}(E, \mathcal{O}(\infty))$ and the infinity point representing the reducible member of the pencil $\bar{s}_{1}+f_{\infty}$ (the curves $\bar{s}_{P_{1}}, \bar{s}_{P_{2}}$ shown on Fig. 4 are members of this pencil). The fact that all the global sections $s \in H^{0}\left(\mathcal{O}_{E}(\infty)\right)$ come from $H^{0}\left(\mathcal{O}_{E}\right)=\{$ constants $\}$ under the embedding $\mathcal{O}_{E} \hookrightarrow \mathcal{O}_{E}(\infty)$ implies that they all vanish at $\infty$. Thus all the $\bar{s} \in\left|\bar{s}_{2}\right|$ pass through the point $f_{\infty} \cdot \bar{s}_{2}$ which is the zero of the fiber of the line bundle $\mathcal{O}_{E}(\infty)$ over $\infty$. 
Using this representation of $S_{0}$, we can prove the existence of a cross-section $\bar{r} \subset S_{0}, \bar{r} \in$ $\left|\bar{s}_{2}+f_{p_{+}}\right|$passing through the three points $P_{0}=\left[p_{+}, v\right]$ and $P_{i}=\left[q_{i}, v_{2}^{(i)}\right](i=1,2)$. Namely, the curves from the linear system $\left|\bar{s}_{2}+f_{p_{+}}\right|$are the sections of $\mathcal{O}_{E}\left(\infty+p_{+}\right)$considered as sections of $\mathcal{O}_{E}(\infty)$ having a simple pole at $p_{+}$. The fact that they have a simple pole at $p_{+}$means that they meet $\bar{s}_{1}$ at $f_{p_{+}} \cdot \bar{s}_{1}$. The vector space $H^{0}\left(\mathcal{O}_{E}\left(\infty+p_{+}\right)\right)$is 2-dimensional, so we can find $r$ in it taking the values $v_{2}^{(1)}, v_{2}^{(2)}$ at $q_{1}$, resp. $q_{2}$.

We have $\bar{s}_{1}^{2}=-1, \bar{s}_{2}^{2}=1, \bar{r}^{2}=3, \bar{s}_{1} \cdot \bar{r}=1$, and $\bar{s}_{1} \cap \bar{r}=P_{0}$. After we perform the 3 elementary transforms at $P_{i}(i=0,1,2)$, the self-intersection $\bar{r}^{2}$ goes down by 3 . At the same time $\bar{s}_{1}^{2}$ goes up by 2 when making elms $P_{1}, P_{2}$ and descends by 1 after the elm at $P_{0}$. Hence in $S^{\prime}=\mathbb{P}\left(\mathcal{E}^{\prime}\right)$, we have two disjoint sections $\bar{r}, \bar{s}_{1}$ with self-intersection 0 . Thus, by Lemma $3, \mathcal{E}^{\prime}=\mathcal{L}_{1} \oplus \mathcal{L}_{2}$, where $\mathcal{L}_{1}, \mathcal{L}_{2}$ are line bundles of the same degree. By Lemma $4, \operatorname{deg} \mathcal{E}^{\prime}=\operatorname{deg} \mathcal{E}+3=0$, hence $\operatorname{deg} \mathcal{L}_{1}=\operatorname{deg} \mathcal{L}_{2}=0$. The direct sum of line bundles of the same degree is strictly semistable.

Next, $\bar{s}_{1}$ (in $S_{0}$ ) corresponds to the line subbundle $\mathcal{O}_{E}(-\infty)$. It remains $\mathcal{O}_{E}(-\infty)$ after elms in $P_{1}, P_{2}$, and becomes $\mathcal{O}_{E}\left(p_{+}-\infty\right)$ after the elm in $P_{0}$. Hence $\mathcal{L}_{1}=\mathcal{O}_{E}\left(p_{+}-\infty\right)$ and $\mathcal{L}_{2}=\operatorname{det} \mathcal{E}^{\prime} \otimes L^{-1}$. But $\operatorname{det} \mathcal{E}^{\prime}=\operatorname{det} \mathcal{E}\left(q_{1}+q_{2}+p_{+}\right)=\mathcal{O}_{E}\left(q_{1}+q_{2}+p_{+}-3 \infty\right)$. Thus $\mathcal{L}_{2}=\mathcal{O}_{E}\left(q_{1}+q_{2}-2 \infty\right)$.

Remark 2. If we fix $E$ and let vary $p_{+}, q_{1}, q_{2}$, then we see that the generic direct sum $\mathcal{L}_{1} \oplus \mathcal{L}_{2}$ of two line bundles of degree 0 occurs as the underlying vector bundle of $\nabla^{\prime}$. According to [17], the moduli space of semistable rank-2 vector bundles on $E$ is isomorphic to the symmetric square $E^{(2)}$ of $E$, and its open set parameterizes, up to an isomorphism, the direct sums $\mathcal{L}_{1} \oplus \mathcal{L}_{2}$. Thus we obtain a natural map from the parameter space of our direct image connections to the symmetric square $E^{(2)}$, whilst using the stable bundles $\mathcal{E}$ of degree -1 provides a natural map onto $E$ (Corollary 3).

Remark 3. Korotkin [12] considers twisted rank-2 connections on $E$ with connection matrices $A$ satisfying the transformation rule

$$
T_{a}(A)=Q A Q^{-1}, \quad T_{b}(A)=R A R^{-1}
$$

for some $2 \times 2$ matrices $Q, R$. In the case when $Q, R$ commute, such a twisted connection can be understood as an ordinary connection on a nontrivial vector bundle $\mathcal{E}$ over $E$ that can be described as follows: let $E=\mathbb{C} / \Lambda$ where $\Lambda$ is the period lattice of $E$ with basis $(1, \tau)$, and let $z$ be the flat coordinate on $E$ (or on the universal cover $\mathbb{C}$ of $E$ ) such that $T_{a}(z)=z+1$, $T_{b}(z)=z+\tau$. Let us make $\Lambda$ act on $\mathbb{C}^{2} \times \mathbb{C}$ by the rule

$$
(v, z) \stackrel{a}{\mapsto}(Q v, z+1), \quad(v, z) \stackrel{b}{\mapsto}(R v, z+\tau) .
$$

Then $\mathcal{E} \rightarrow E$ is obtained as the quotient $\mathbb{C}^{2} \times \mathbb{C} / \Lambda \rightarrow \mathbb{C} / \Lambda$ of the trivial vector bundle $\mathbb{C}^{2} \times \mathbb{C} \stackrel{p r_{2}}{\longrightarrow} \mathbb{C}$.

However, the twisted connections obtained in [12] satisfy (20) with non-commuting $Q, R$, given by Pauli matrices:

$$
Q=\sigma_{1}=\left(\begin{array}{cc}
0 & 1 \\
1 & 0
\end{array}\right), \quad R=\sigma_{3}=\left(\begin{array}{cc}
1 & 0 \\
0 & -1
\end{array}\right) .
$$

This follows from the relation $A=\mathrm{d} \Psi \Psi^{-1}$, where $\Psi$ is a fundamental matrix of the connection, and from the transformation law for $\Psi: T_{a}(\Psi)=i \sigma_{1} \Psi, T_{b}(\Psi)=i \sigma_{3} \Psi e^{-2 i \pi \lambda \sigma_{3}}$, where $\lambda \in \mathbb{C}$ is a parameter (see (3.74) in loc. cit). Hence Korotkin's connections are really twisted and have no underlying vector bundles. This is a major difference between the result of [12] and that of the present paper. Another difference, concerning the method, is that the starting point in [12] is an ad hoc expression for $\Psi$ in terms of Prym theta functions of the double cover $C \rightarrow E$, and the connection matrix is implicit. 
F.-X. Machu

\section{Monodromy and differential Galois groups}

Let $G$ be the monodromy group of the connection $\nabla_{\mathcal{E}}$ on $f_{*} \mathcal{L}=\mathcal{E}$ defined by formula (12). It is the subgroup of $G L(2, \mathbb{C})$ generated by $M_{a}, M_{b}, M_{\gamma_{1}}$. We will first consider the case of generic values of the parameters $\left(\lambda_{1} K, \lambda_{1} L, \lambda_{2} K^{\prime}, \lambda_{2} L^{\prime}\right)$. Here, generic means that the point belongs to the complement of a countable union of affine $\mathbb{Q}$-subspaces of $\mathbb{C}^{4}$. More exactly, we require that the triples $\left(i \lambda_{2} K^{\prime}, \lambda_{2} L^{\prime}, i \pi\right)$ and $\left(\lambda_{1} K, i \lambda_{1} L, \pi\right)$ are free over $\mathbb{Q}$. Let

$$
R^{\theta}=\left(\begin{array}{cc}
\cos \theta & i \sin \theta \\
i \sin \theta & \cos \theta
\end{array}\right), \quad H^{\theta}=\left(\begin{array}{cc}
\cosh \theta & \sinh \theta \\
\sinh \theta & \cosh \theta
\end{array}\right) \quad\left(H^{i \theta}=R^{\theta}\right) .
$$

Let $N$ be the normal subgroup of $G$ defined by

$$
N=\{X \in G \mid \operatorname{det} X= \pm 1\} .
$$

We have:

$$
N=\left\{\prod_{i=1}^{r} M_{a}^{j_{i}} M_{b}^{k_{i}} M_{\gamma_{1}}^{\epsilon_{i}} \mid r \geq 0, j_{i} \in \mathbb{Z}, k_{i} \in \mathbb{Z}, \epsilon_{i} \in\{0,1\}, \sum_{i} k_{i}=\sum_{i} j_{i}=0\right\} .
$$

We can write $G$ as the semi-direct product of $N$ with the subgroup of $G$ generated by $M_{a}, M_{b}$. The latter is identified with $\mathbb{Z} \times \mathbb{Z}$, so $G=N \rtimes(\mathbb{Z} \times \mathbb{Z})$. Let $N_{1}$ be the subgroup of $N$ generated by $R^{4 \lambda_{1} K}, H^{4 \lambda_{1} L}$. As $M_{a}=e^{-2 i \lambda_{2} K^{\prime}} R^{-2 \lambda_{1} K}$, and $M_{b}=e^{2 \lambda_{2} L^{\prime}} H^{2 \lambda_{1} L}$, we have $\left[M_{a}, M_{\gamma_{1}}\right]=R^{-4 \lambda_{1} K}$, $\left[M_{b}, M_{\gamma_{1}}\right]=H^{4 \lambda_{1} L},\left[M_{a}, M_{b}\right]=1$. Hence $N$ is the semi-direct product $N=N_{1} \rtimes \mu_{2}$, where $\mu_{n} \simeq \mathbb{Z} / n \mathbb{Z}$ denotes a cyclic group of order $n$, and the factor $\mu_{2}$ of the semi-direct product is generated by $M_{\gamma_{1}}$. Finally, we obtain a normal sequence $1 \triangleleft N_{1} \triangleleft N \triangleleft G$ with successive quotients $\mathbb{Z} \times \mathbb{Z}, \mathbb{Z} / 2 \mathbb{Z}, \mathbb{Z} \times \mathbb{Z}$, all of whose levels are semi-direct products. We can write:

$$
G \simeq((\mathbb{Z} \times \mathbb{Z}) \rtimes \mathbb{Z} / 2 \mathbb{Z}) \rtimes(\mathbb{Z} \times \mathbb{Z}) .
$$

We have also $N_{1}=D(G)$, the commutator subgroup of $G$. As $D(G) \simeq \mathbb{Z} \times \mathbb{Z}$ is Abelian, $G$ is solvable of height 2 .

From now on, we go over to the general case. The formulas (21), (22) are no more equivalent. Let us define $N$ by (22), and $N_{1}$ by the same formula with the additional condition $\sum_{i} \epsilon_{i} \equiv 0(2)$. We have again the normal sequence $1 \triangleleft N_{1} \triangleleft N \triangleleft G$. Its first level is a semidirect product, $N=N_{1} \rtimes \mu_{2}$, but the upper one may be a nonsplit extension. Define two group epimorphisms

$$
\begin{array}{ll}
\mathbb{Z} \times \mathbb{Z} \stackrel{\varphi_{1}}{\longrightarrow} N_{1}, & \mathbb{Z} \times \mathbb{Z} \stackrel{\varphi_{2}}{\longrightarrow} G / N, \\
\left(n_{1}, n_{2}\right) \longmapsto \frac{\sigma\left(n_{1}, n_{2}\right)^{2}}{\operatorname{det} \sigma\left(n_{1}, n_{2}\right)}, & \left(n_{1}, n_{2}\right) \longmapsto \sigma\left(n_{1}, n_{2}\right) N,
\end{array}
$$

where $\sigma\left(n_{1}, n_{2}\right)=M_{a}^{n_{1}} M_{b}^{n_{2}}$.

Thus both $N_{1}$ and $G / N$ are quotients of $\mathbb{Z} \times \mathbb{Z}$. We want to find out, which pairs $Q_{1}, Q_{2}$ of quotients of $\mathbb{Z} \times \mathbb{Z}$ can be realized as the pair $N_{1}, G / N$ for some monodromy group $G$. We will denote by $\pi_{N}$ the canonical epimorphism $G \rightarrow G / N$, and the maps $\bar{\varphi}_{1}, \bar{\varphi}_{2}$ are defined by the following commutative diagram:

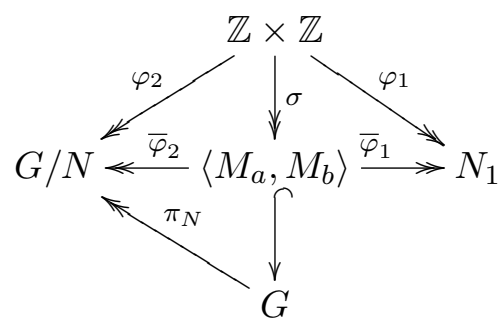


One can also give $\bar{\varphi}_{1}$ by the formulas

$$
\bar{\varphi}_{1}(X)=\frac{1}{\operatorname{det} X} X^{2}=\left[X, M_{\gamma_{1}}\right] \quad \text { for all } X \in\left\langle M_{a}, M_{b}\right\rangle \text {. }
$$

Proposition 15. For any connection (12), its monodromy group $G$ fits into a normal sequence $N_{1} \triangleleft N \triangleleft G$ in such a way, that the following properties are verified:

1. Both $N_{1}$ and $G / N$ are quotients of $\mathbb{Z} \times \mathbb{Z}$, and $N / N_{1} \simeq \mu_{2}$.

2. The extension $N_{1} \triangleleft N$ is always split: $N \simeq N_{1} \rtimes \mu_{2}$, the generator $h \in \mu_{2}$ acting on $N_{1}$ via the map $g \mapsto g^{-1}$.

3. The subgroup $\left\langle M_{a}, M_{b}\right\rangle$ of $G$ provides a splitting of the extension $N \triangleleft G$ if and only if $\bar{\varphi}_{2}$ is an isomorphism. In this case, the action of $G / N$ on $N$ defining the split extension is given by $x: g \mapsto g$ and $x: h \mapsto \bar{\varphi}_{1} \bar{\varphi}_{2}^{-1}(x) h$ for any $x \in G / N, g \in N_{1}$.

Conversely, let $\left(Q_{1}, Q_{2}\right)$ be a pair of group quotients of $\mathbb{Z} \times \mathbb{Z}$. Then $\left(Q_{1}, Q_{2}\right)$ can be realized as the pair $\left(N_{1}, G / N\right)$ for the monodromy group $G$ of a connection (12) if and only if $\left(Q_{1}, Q_{2}\right)$ occurs in the following table:

\begin{tabular}{|c|c|c|c|c|c|}
\hline $\mathrm{N}^{\circ}$ & rk $Q_{1}$ & rk $Q_{2}$ & $Q_{1}$ & $Q_{2}$ & Restrictions \\
\hline $1^{*}$ & 2 & 2 & $\mathbb{Z} \times \mathbb{Z}$ & $\mathbb{Z} \times \mathbb{Z}$ & - \\
2 & 2 & 1 & $\mathbb{Z} \times \mathbb{Z}$ & $\mu_{d} \times \mathbb{Z}$ & $2 \mid d$ \\
3 & 2 & 0 & $\mathbb{Z} \times \mathbb{Z}$ & $\mu_{2} \times \mu_{d}$ & $2 \mid d$ \\
\hline $4^{*}$ & 1 & 2 & $\mu_{d} \times \mathbb{Z}$ & $\mathbb{Z} \times \mathbb{Z}$ & $d \geq 1$ \\
5 & 1 & 1 & $\mu_{d} \times \mathbb{Z}$ & $\mu_{d^{\prime}} \times \mathbb{Z}$ & if $2 \mid d$, then $2 \mid d^{\prime}$ \\
6 & 1 & 0 & $\mu_{d} \times \mathbb{Z}$ & $\mu_{d^{\prime}}$ & $2 \nmid d, 2 \mid d^{\prime}$ \\
7 & 1 & 0 & $\mu_{d} \times \mathbb{Z}$ & $\mu_{2} \times \mu_{d^{\prime}}$ & $d \geq 1,2 \mid d^{\prime}$ \\
\hline $8^{*}$ & 0 & 2 & $\mu_{d}$ & $\mathbb{Z} \times \mathbb{Z}$ & $d \geq 1$ \\
9 & 0 & 1 & $\mu_{d}$ & $\mu_{d^{\prime}} \times \mathbb{Z}$ & $d \equiv d^{\prime} \bmod 2$ \\
10 & 0 & 0 & $\mu_{d}$ & $\mu_{d^{\prime}}$ & $d \geq 1, d^{\prime} \geq 1$ \\
11 & 0 & 0 & $\mu_{d}$ & $\mu_{2} \times \mu_{d^{\prime}}$ & $2|d, 2| d^{\prime}$ \\
\hline
\end{tabular}

The items whose numbers are marked with an asterisk correspond to the pairs that always give a split extension $N \triangleleft G$.

Proof. The first part, resuming the properties of the tower of group extensions $N_{1} \triangleleft N \triangleleft G$, is an easy exercise, and we go over to the second one. Given a pair $\left(Q_{1}, Q_{2}\right)$, we find out whether it is possible to choose epimorphisms $\mathbb{Z} \times \mathbb{Z} \stackrel{\varphi_{1}}{\longrightarrow} Q_{1}$ and $\mathbb{Z} \times \mathbb{Z} \stackrel{\varphi_{2}}{\longrightarrow} Q_{2}$ and identify them as the morphisms defined in (23) for a suitable choice of matrices $M_{a}, M_{b}$. The proof follows a case by case enumeration of different types of kernels of $\varphi_{1}$ and $\varphi_{2}$. To shorten the notation, let us write $M_{a}=e^{\alpha_{1}} H^{\beta_{1}}, M_{b}=e^{\alpha_{2}} H^{\beta_{2}}$. The case $\operatorname{rk} \operatorname{ker} \varphi_{1}=\operatorname{rk} \operatorname{ker} \varphi_{2}=0$, corresponding to $\operatorname{rk}_{\mathbb{Q}}\left(\alpha_{1}, \beta_{1}, \pi i\right)=\operatorname{rk}_{\mathbb{Q}}\left(\alpha_{2}, \beta_{2}, \pi i\right)=3$, has been treated before the statement of the proposition. It gives item 1 of the table.

The proofs of all the other cases resemble each other, and we will give only one example of this type of argument, say, when both kernels are of rank 1. Under this assumption, there exist $\left(d, k_{1}, k_{2}\right) \in \mathbb{Z}^{3}$ and $\left(d^{\prime}, k_{1}^{\prime}, k_{2}^{\prime}\right) \in \mathbb{Z}^{3}$ such that

$$
d \geq 1, \quad d^{\prime} \geq 1, \quad \operatorname{gcd}\left(k_{1}, k_{2}\right)=1, \quad \operatorname{gcd}\left(k_{1}^{\prime}, k_{2}^{\prime}\right)=1,
$$

and

$$
\operatorname{ker} \varphi_{1}=\left\langle d\left(k_{1}, k_{2}\right)\right\rangle, \quad \operatorname{ker} \varphi_{2}=\left\langle d^{\prime}\left(k_{1}^{\prime}, k_{2}^{\prime}\right)\right\rangle \text {. }
$$


For $\left(n_{1}, n_{2}\right) \in \mathbb{Z}^{2}$, we have:

$$
\begin{aligned}
& \left(n_{1}, n_{2}\right) \in \operatorname{ker} \varphi_{1} \Longleftrightarrow \exists m \in \mathbb{Z} \mid n_{1} \beta_{1}+n_{2} \beta_{2}=\pi i m ; \\
& \left(n_{1}, n_{2}\right) \in \operatorname{ker} \varphi_{2} \Longleftrightarrow \exists\left(m_{1}, m_{2}\right) \in \mathbb{Z}^{2} \mid M_{a}^{n_{1}} M_{b}^{n_{2}}=\left(H^{2 \beta_{1}}\right)^{m_{1}}\left(H^{2 \beta_{2}}\right)^{m_{2}} .
\end{aligned}
$$

The latter equality can be written in the form $e^{\alpha} H^{\beta}=1$, where

$$
\alpha=n_{1} \alpha_{1}+n_{2} \alpha_{2}, \quad \beta=\left(n_{1}-2 m_{1}\right) \beta_{1}+\left(n_{2}-2 m_{2}\right) \beta_{2} .
$$

As $e^{\alpha} H^{\beta}=1$ if and only if $e^{\alpha}=H^{\beta}= \pm 1$, we see that the condition of (26) is equivalent to the existence of an integer vector $\left(m_{0}, m_{1}, m_{2}, m_{3}\right) \in \mathbb{Z}^{4}$ such that

$$
\begin{aligned}
& m_{0} \equiv m_{3} \bmod 2 \\
& n_{1} \alpha_{1}+n_{2} \alpha_{2}=\pi i m_{0}, \\
& \left(n_{1}-2 m_{1}\right) \beta_{1}+\left(n_{2}-2 m_{2}\right) \beta_{2}=\pi i m_{3} .
\end{aligned}
$$

Substituting the generators of $\operatorname{ker} \varphi_{i}$ for $\left(n_{1}, n_{2}\right)$, we obtain the following system of equations:

$$
\begin{aligned}
& d k_{1} \beta_{1}+d k_{2} \beta_{2}=\pi i m, \\
& d^{\prime} k_{1}^{\prime} \alpha_{1}+d^{\prime} k_{2}^{\prime} \alpha_{2}=\pi i m_{0}, \\
& \left(d^{\prime} k_{1}^{\prime}-2 m_{1}\right) \beta_{1}+\left(d^{\prime} k_{2}^{\prime}-2 m_{2}\right) \beta_{2}=\pi i m_{3} .
\end{aligned}
$$

The condition that $d\left(k_{1}, k_{2}\right), d^{\prime}\left(k_{1}^{\prime}, k_{2}^{\prime}\right)$ are not just elements of the corresponding kernels, but their generators, is transcribed as follows:

$$
\operatorname{gcd}(m, d)=\operatorname{gcd}\left(d^{\prime}, m_{0}, 2 m_{1}, 2 m_{2}, m_{3}\right)=1
$$

for any $\left(m_{1}, m_{2}, m_{3}\right)$ satisfying $(27),(32)$.

As rk $\operatorname{ker} \varphi_{1}=1$, the equations (30) and (32) have to be proportional. If $d^{\prime}$ is odd, but $d$ is even, then at least one of the coefficients of $\beta_{i}$ in (32) is odd. But both coefficients in (30) are even, and this contradicts (33). We get the restriction from item 5 of the table: if $d$ is even, then $d^{\prime}$ is even, too. This leaves possible three combinations of parities of $d, d^{\prime}$, and it is easy to see that a solution to (24), (30)-(33) exists for any of them. For example, if $d \equiv d^{\prime} \bmod 2$, then we can choose $k_{i}, k_{i}^{\prime}$ in such a way that $k_{i} \equiv k_{i}^{\prime} \bmod 2(i=1,2), k_{1} k_{1}^{\prime} \neq 0$. We get a solution to the problem as follows:

$$
\begin{array}{ll}
m_{i} & =\frac{1}{2} d\left(k_{i}^{\prime}-k_{i}\right) \quad(i=1,2), \quad m=m_{0}=m_{3}=1, \quad \alpha_{2}=\beta_{2}=1, \\
\alpha_{1} & =\frac{\pi i-d^{\prime} k_{2}^{\prime}}{d^{\prime} k_{1}^{\prime}}, \quad \beta_{1}=\frac{\pi i-d k_{2}}{d k_{1}} .
\end{array}
$$

Our choice for $\alpha_{2}, \beta_{2}$ is explained by the observation that we should have $\operatorname{rk}_{\mathbb{Q}}\left(\alpha_{1}, \beta_{1}, \pi i\right)=$ $\operatorname{rk}_{\mathbb{Q}}\left(\alpha_{2}, \beta_{2}, \pi i\right)=2$, and 1 is the simplest complex number which is not a rational multiple of $\pi i$.

Remark 4. In the above proof, if $\operatorname{ker} \varphi_{2} \not \subset \operatorname{ker} \varphi_{1}$, then any solution of (30)-(32) satisfies the condition $\left(m_{1}, m_{2}\right) \neq 0$, which means that $d^{\prime}\left(k_{1}^{\prime}, k_{2}^{\prime}\right) \notin \operatorname{ker} \sigma$. Hence $\sigma\left(d^{\prime}\left(k_{1}^{\prime}, k_{2}^{\prime}\right)\right)$ is a nonzero element of $\operatorname{ker} \bar{\varphi}_{2}$, and $\bar{\varphi}_{2}$ is not an isomorphism. This implies that the extension $N \triangleleft G$ is nonsplit. Hence it is never split, unless $d \mid d^{\prime}$. In this case, it can be occasionally split, if $\operatorname{ker} \varphi_{2} \subset \operatorname{ker} \varphi_{1}$.

We can deduce from Proposition 15 a description of all the finite monodromy groups; they correspond to lines 10 and 11 of the table. This description is only partial, because we do not determine completely the extension data. 
Corollary 4. All the finite monodromy groups $G$ of connections (12) are obtained as extensions

$$
D_{d} \hookrightarrow G \rightarrow \mu_{d^{\prime}} \quad\left(d \geq 1, d^{\prime} \geq 1\right)
$$

or

$$
D_{d} \hookrightarrow G \rightarrow \mu_{2} \times \mu_{d^{\prime}} \quad\left(2|d, 2| d^{\prime}\right),
$$

where $D_{d}=\mu_{d} \rtimes \mu_{2}$ is the dihedral group.

Corollary 5. The only finite Abelian groups occurring as the monodromy groups of connections (12) are $\mu_{2}$ and $\mu_{2} \times \mu_{d}(d \geq 2)$.

We add a few examples of infinite monodromy groups with nongeneric parameters $\left(\lambda_{1} K, \lambda_{1} L\right.$, $\left.\lambda_{2} K^{\prime}, \lambda_{2} L^{\prime}\right)$.

Example 1. It is easy to select the parameters to get for $G$ one of the groups $D_{n} \times \mathbb{Z}^{i}$ or $D_{n} \rtimes \mathbb{Z}^{i}$, where $n \in \mathbb{N} \cup\{\infty\}, i=0,1,2$. For example, to get $D_{n} \rtimes \mathbb{Z}$, we can set $M_{a}=R^{\frac{2 \pi}{n}}$, $M_{b}=R^{1}$, and to get $D_{n} \times \mathbb{Z}$, we can set $M_{a}=R^{\frac{2 \pi}{n}}, M_{b}=1$.

Now that we have described the structure of the monodromy group of $\nabla_{\mathcal{E}}$, we can ask the question on its Zariski closure. According to [10, Proposition 5.2], the Zariski closure of $G$ is the differential Galois group $\operatorname{DGal}\left(\nabla_{\mathcal{E}}\right)$. For the reader's convenience, we recall its definition.

Let $\left(K,,^{\prime}\right)$ be a differential field with field of constants $\mathbb{C}$. This means that $K$ is endowed with a $\mathbb{C}$-linear derivation ' $: K \rightarrow K$.

Definition 6. Let $\left(K,{ }^{\prime}\right) \subset\left(L,,^{\prime}\right)$ be an extension of differential fields with field of constants $\mathbb{C}$. The differential Galois group $\operatorname{DGal}(L / K)$ is the group consisting of all the $K$-automorphisms $\sigma$ of $L$ such that $\sigma\left(f^{\prime}\right)=(\sigma(f))^{\prime}$ for all $f \in L$.

If $L$ is finitely generated as a $K$-algebra, say, by $p$ elements, then $\operatorname{DGal}(L / K)$ can be embedded onto $G L(p, \mathbb{C})$, and it is an algebraic group if considered as a subgroup of $G L(p, \mathbb{C})$ in this embedding.

We apply this definition to $K=\mathbb{C}(E)$, the derivation ' being the differentiation with respect to some nonconstant function $z \in K$. Given a connection $\nabla_{\mathcal{E}}$ on $E$, we can consider a fundamental matrix $\Phi$ of its solutions, and set $L$ to be the field generated by all the matrix elements of $\Phi$. The group $\operatorname{DGal}\left(\nabla_{\mathcal{E}}\right)$ is defined to be $\operatorname{DGal}(L / K)$. See $[18,19]$ for more details.

Remark that the monodromy group $G$ lies in the subgroup $\mathbb{G}$ of $G L(2, \mathbb{C})$ defined by

$$
\mathbb{G}=\left\{\left(\begin{array}{ll}
C \alpha & C \epsilon \beta \\
C \beta & C \alpha \epsilon
\end{array}\right) \mid C \in \mathbb{C}^{*},(\alpha, \beta) \in \mathbb{C}^{2}, \epsilon \in\{-1,1\}, \alpha^{2}-\beta^{2}=1\right\} .
$$

Denote by $\mathbb{G}_{0}$ the connected component of unity in $\mathbb{G}$, singled out by the condition $\epsilon=1$. The Zariski closure $\bar{G}$ of $G$ is contained in $\mathbb{G}$ and is not contained in $\mathbb{G}_{0}$. The following statement is obvious:

Lemma 8. Let $\psi: \mathbb{C}^{*} \times \mathbb{C}^{*} \rtimes\{-1,1\} \longrightarrow \mathbb{G}$ be defined by

$$
(\lambda, \mu, \epsilon) \longmapsto\left(\begin{array}{cc}
\lambda \alpha & \lambda \beta \epsilon \\
\lambda \beta & \lambda \alpha \epsilon
\end{array}\right)
$$

with $\alpha=\frac{1}{2}\left(\mu+\frac{1}{\mu}\right), \beta=\frac{1}{2}\left(\mu-\frac{1}{\mu}\right)$. Then $\psi$ is a surjective morphism with kernel $\{(1,1,1)$, $(-1,-1,-1)\}$. 
We see that $\mathbb{G}_{0}=\psi\left(\mathbb{C}^{*} \times \mathbb{C}^{*}\right)$ is identified with the quotient $\mathbb{C}^{*} \times \mathbb{C}^{*} /\{-1,1\}$, and the latter is isomorphic to $\mathbb{C}^{*} \times \mathbb{C}^{*}$ via the map $\left(z_{1}, z_{2}\right) \bmod \{-1,1\} \longmapsto\left(z_{1} z_{2}, \frac{z_{1}}{z_{2}}\right)$. Thus we get an explicit isomorphism $\mathbb{G}_{0} \simeq \mathbb{C}^{*} \times \mathbb{C}^{*}$. Using this identification, one can easily determine the Zariski closure $\overline{G_{0}}$ of the subgroup $G_{0}=G \cap \mathbb{G}_{0}=\left\langle M_{a}, M_{b}\right\rangle$ of $\mathbb{C}^{*} \times \mathbb{C}^{*}$, and $\operatorname{DGal}\left(\nabla_{\mathcal{E}}\right)=\overline{G_{0}} \rtimes\left\langle M_{\gamma_{1}}\right\rangle$.

We can use the following observations:

a) If a pair $(s, t) \in \mathbb{C}^{*} \times \mathbb{C}^{*}$ is such that $\mathrm{rk}_{\mathbb{Q}}(\ln (s), \ln (t), i \pi)=1$ (that is, $s$ and $t$ are roots of unity), then the group generated by the pair $(s, t)$ is finite and coincides with its closure.

b) If a pair $(s, t) \in \mathbb{C}^{*} \times \mathbb{C}^{*}$ is such that $\mathrm{rk}_{\mathbb{Q}}(\ln (s), \ln (t), i \pi)=2$, and $k_{1} \ln (s)+k_{2} \ln (t)+2 k_{3} i \pi=$ 0 is a $\mathbb{Z}$-linear relation with relatively prime $k_{i}$, then $\overline{\langle(s, t)\rangle}$ is the subgroup $V$ of $\mathbb{C}^{*} \times \mathbb{C}^{*}$ defined by $z_{1}^{k_{1}} z_{2}^{k_{2}}=1$, isomorphic to $\mathbb{C}^{*} \times \mu_{d}$, where $d=\operatorname{gcd}\left(k_{1}, k_{2}\right)$, and $\mu_{d}$ is the cyclic group of order $d$.

c) If the triple $(\ln (s), \ln (t), \pi i)$ is free over $\mathbb{Q}$, then the closure of $\langle(s, t)\rangle$ is $\mathbb{C}^{*} \times \mathbb{C}^{*}$.

Apply this to pairs $(s, t)$ belonging to the subgroup generated by two pairs $\left(s_{1}, t_{1}\right),\left(s_{2}, t_{2}\right)$ which are the images of $M_{a}$, resp. $M_{b}$. Then if $\left(\ln \left(s_{j}\right), \ln \left(t_{j}\right), \pi i\right)$ is free over $\mathbb{Q}$ for at least one value of $j=1$ or 2 , then $\overline{G_{0}}=\mathbb{C}^{*} \times \mathbb{C}^{*}$ and $\operatorname{DGal}\left(\nabla_{\mathcal{E}}\right)=\mathbb{G}$. In the case when both triples $\left(\ln \left(s_{1}\right), \ln \left(t_{1}\right), \pi i\right),\left(\ln \left(s_{2}\right), \ln \left(t_{2}\right), \pi i\right)$ are not free over $\mathbb{Q}$, the necessary and sufficient condition for $\overline{\left\langle\left(s_{1}, t_{1}\right),\left(s_{1}, s_{2}\right)\right\rangle}$ to be $\mathbb{C}^{*} \times \mathbb{C}^{*}$ is the following: $\operatorname{rk}_{\mathbb{Q}}\left(\ln \left(s_{j}\right), \ln \left(t_{j}\right), i \pi\right)=2$ for both values $j=1,2$, and if $a_{j 1} \ln \left(s_{j}\right)+a_{j 2} \ln \left(t_{j}\right)+a_{j 3} \pi i=0(j=1,2)$ are nontrivial $\mathbb{Q}$-linear relations in

these triples, then $\left|\begin{array}{ll}a_{11} & a_{12} \\ a_{21} & a_{22}\end{array}\right| \neq 0$. This condition can be easily formulated in terms of the epimorphisms $\varphi_{i}$ defined in (23): $\operatorname{ker} \varphi_{1}, \operatorname{ker} \varphi_{2}$ are both of rank 1 and $\operatorname{ker} \varphi_{1} \cap \operatorname{ker} \varphi_{2}=0$. In this case we have the same conclusion: $\operatorname{DGal}\left(\nabla_{\mathcal{E}}\right)=\mathbb{G}$.

We obtain the following description of possible differential Galois groups of connections (12):

Proposition 16. Let $r_{i}=\operatorname{rk}_{\mathbb{Q}} \operatorname{ker} \varphi_{i}(i=1,2)$.

(i) $\operatorname{DGal}\left(\nabla_{\mathcal{E}}\right)=\mathbb{G}$ if and only if one of the following condition is verified: either $\min \left\{r_{1}, r_{2}\right\}=$ 0 , or $r_{1}=r_{2}=1$ and $\operatorname{ker} \varphi_{1} \cap \operatorname{ker} \varphi_{2}=0$.

(ii) $\operatorname{DGal}\left(\nabla_{\mathcal{E}}\right)$ is a 1-dimensional subgroup of $\mathbb{G}$ if and only if $\min \left\{r_{1}, r_{2}\right\}=1$ and the condition of (i) is not satisfied. Then there exists a one-parameter subgroup $V_{0}$ and a finite cyclic subgroup $\mu_{d}$ in $\mathbb{G}$ such that $\mathrm{DGal}\left(\nabla_{\mathcal{E}}\right)=\left(V_{0} \mu_{d}\right) \rtimes\left\langle M_{\gamma_{1}}\right\rangle$.

(iii) $\operatorname{DGal}\left(\nabla_{\mathcal{E}}\right)$ is finite if and only if $r_{1}=r_{2}=2$, and then $\operatorname{DGal}\left(\nabla_{\mathcal{E}}\right)=G$.

\section{Acknowledgements}

I am greatly indebted to my research advisor D. Markushevich for his encouragement and help. I would like to thank D. Korotkin for explaining me some points. I also acknowledge with pleasure the hospitality of the Mathematics Institute of the Chinese Academy of Sciences, where was done a part of the work on the article. The work was partially supported by the Conseil Departemental du Nord.

\section{References}

[1] Anosov D.A., Bolibruch A.A., The Riemann-Hilbert problem, Aspects of Mathematics, Vol. 22, Vieweg Verlag, 1994.

[2] Atiyah M.F., Vector bundles over an elliptic curve, Proc. London Math. Soc. (3) 7 (1957), 414-452.

[3] Deligne P., Equations différentielles à points singuliers réguliers, Lecture Notes in Math., Vol. 163, Springer Verlag, 1970. 
[4] Diem C., Families of elliptic curves with genus 2 covers of degree 2, Collect. Math. 57 (2006), 1-25, math.AG/0312413.

[5] Enolski V.Z., Grava T., Singular $Z_{N}$ curves, Riemann-Hilbert problem and modular solutions of the Schlesinger equations, Int. Math. Res. Not. 2004 (2004), no. 32, 1619-1683, math-ph/0306050.

[6] Esnault H., Viehweg E., Logarithmic de Rham complexes and vanishing theorems, Invent. Math. 86 (1986), no. $1,161-194$.

[7] Esnault H., Viehweg E., Semistable bundles on curves and irreducible representations of the fundamental group, in Algebraic Geometry, Hirzebruch 70 (Warsaw, 1998), Contemp. Math. 241 (1999), 129-138, math.AG/9808001.

[8] Griffiths P.H., Harris J., Principles of Algebraic Geometry, John Wiley \& Sons, New York, 1978.

[9] Jacobi C., Review of Legendre, Théorie des fonctions elliptiques, Troisième supplément, J. Reine Angew. Math. 8 (1832), 413-417.

[10] Katz M.N., A conjecture in the arithmetic theory of differential equations, Bull. Soc. Math. France 110 (1982), 203-239.

[11] Mumford D., Prym varieties. I. Contributions to analysis, in A Collection of papers dedicated to Lipman Bers, Academic Press, New York, 1974, 325-350.

[12] Korotkin D.A., Isomonodromic deformations in genus zero and one: algebrogeometric solutions and Schlesinger transformations. Integrable systems: from classical to quantum, CRM Proc. Lecture Notes, Vol. 26, Amer. Math. Soc, Providence, RI, 2000, 87-104.

[13] Korotkin D.A., Solution of matrix Riemann-Hilbert problems with quasi-permutation monodromy matrices, Math. Ann. 329 (2004), 335-364, math-ph/0306061.

[14] Lange H., Narasimhan M.S., Maximal subbundles of rank two vector bundles on curves, Math. Ann. 266 (1983), 55-72.

[15] Loray F., van der Put M., Ulmer F., The Lamé family of connections on the projective line, ccsd-00005796.

[16] Shaska T., Völklein H., Elliptic subfields and automorphisms of genus 2 function fields, in Algebra, Arithmetic and Geometry with Applications (West Lafayette, IN, 2000), Springer, Berlin, 2004, 703-723.

[17] Tu L.W., Semistable bundles over an elliptic curve, Adv. Math. 98 (1993), no. 11, 1-26.

[18] van der Put M., Galois theory of differential equations, algebraic groups and Lie algebras, in Differential Algebra and Differential Equations, J. Symbolic Comput. 28 (1999), 441-472.

[19] van der Put M., Singer F., Galois theory of linear differential equations, Springer-Verlag, 2003. 